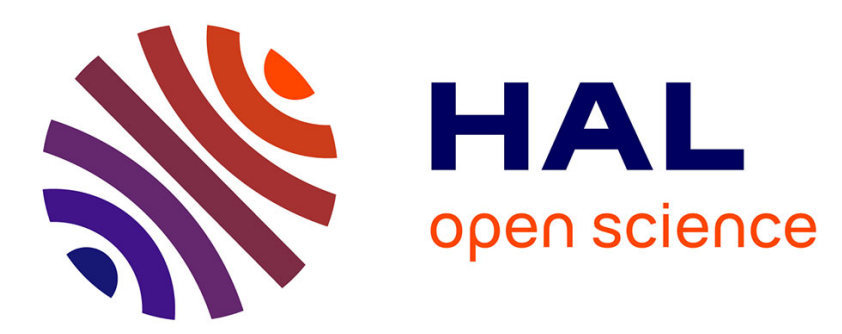

\title{
A hierarchy of dispersive layer-averaged approximations of Euler equations for free surface flows
}

\author{
Enrique D. Fernandez-Nieto, Martin Parisot, Yohan Penel, Jacques
}

Sainte-Marie

\section{- To cite this version:}

Enrique D. Fernandez-Nieto, Martin Parisot, Yohan Penel, Jacques Sainte-Marie. A hierarchy of dispersive layer-averaged approximations of Euler equations for free surface flows. Communications in Mathematical Sciences, 2018, 16 (5), pp.1169-1202. 10.4310/CMS.2018.v16.n5.a1 . hal-01324012v4

\section{HAL Id: hal-01324012 \\ https://hal.science/hal-01324012v4}

Submitted on 1 Feb 2018

HAL is a multi-disciplinary open access archive for the deposit and dissemination of scientific research documents, whether they are published or not. The documents may come from teaching and research institutions in France or abroad, or from public or private research centers.
L'archive ouverte pluridisciplinaire HAL, est destinée au dépôt et à la diffusion de documents scientifiques de niveau recherche, publiés ou non, émanant des établissements d'enseignement et de recherche français ou étrangers, des laboratoires publics ou privés. 


\title{
A hierarchy of dispersive layer-averaged approximations of Euler equations for free surface flows
}

\author{
E.D. Fernández-Nieto ${ }^{1}$, M. Parisot ${ }^{2}$, Y. Penel$^{2}$, and J. Sainte-Marie ${ }^{2}$ \\ ${ }^{1}$ Dpto. Matemática Aplicada I. ETS Arquitectura, Universidad de Sevilla, \\ Avda Reina Mercedes N. 2, 41012 Sevilla, Spain \\ ${ }^{2}$ Team ANGE (Inria, CEREMA, UPMC, CNRS), \\ 2 rue Simone Iff, CS 42112, 75589 Paris Cedex 12, France \\ and Sorbonne Universités, UPMC Univ. Paris 06, \\ Lab. Jacques-Louis Lions UMR CNRS 7598, 75005 Paris, France
}

February 1, 2018

\begin{abstract}
In geophysics, the shallow water model is a good approximation of the incompressible Navier-Stokes system with free surface and it is widely used for its mathematical structure and its computational efficiency. However, applications of this model are restricted by two approximations under which it was derived, namely the hydrostatic pressure and the vertical averaging. Each approximation has been addressed separately in the literature: the first one was overcome by taking into account the hydrodynamic pressure (e.g. the non-hydrostatic or the Green-Naghdi models); the second one by proposing a multilayer version of the shallow water model.

In the present paper, a hierarchy of new models is derived with a layerwise approach incorporating non-hydrostatic effects to approximate the Euler equations. To assess these models, we use a rigorous derivation process based on a Galerkin-type approximation along the vertical axis of the velocity field and the pressure, it is also proven that all of them satisfy an energy equality. In addition, we analyse the linear dispersion relation of these models and prove that the latter relations converge to the dispersion relation for the Euler equations when the number of layers goes to infinity.
\end{abstract}

\section{Introduction}

Water is present all around the world and as such it is of major interest for societies whether it be in the purpose of producing energy (tides, swell, currents, ...) or in order to protect people from hazardous flows. That is why the management of water resources is extensively investigated in the literature. To predict the evolution of lakes, rivers or oceans, scientists derive simplified models with a balance between physical relevance (matching with experimental or actual results) and computational efficiency (running costs).

Despite the outstanding enhancement of computer performance, it is still a great issue to simulate the complete set of equations governing fluid flows like the free-surface compressible Navier-Stokes equations. Both theoretical and numerical issues related to the modelling of free-surface flows by means of these equations may be prohibitive (in particular because the surface is an unknown itself) so that models of reduced complexity were derived relying on simplifying assumptions (incompressible, inviscid, irrotational, shallow, ... ). Such simplifications - which allowed to neglect some terms in the equations - may arise at the expense of physics since some phenomena are not taken into account anymore. However, the influence of the simplifying process is sometimes hard to determine if no exact solution is available. In the sequel, we shall focus on incompressible inviscid free-surface flows governed by the Euler equations. 
Among the simplifying assumptions justifying the approximation of the Euler system by the Shallow Water equations [22,27], two of them seem restrictive in several situations, typically the propagation of waves in coastal areas. The first one consists in focusing on the so-called hydrostatic pressure which implies to neglect the vertical acceleration of the fluid and hence some of the dispersive effects. The second one is the vertical averaging which amounts to only considering the main current, i.e. the vertical average of the current. Several works are aimed at circumventing one or the other of these hypotheses (a brief state of art is proposed in $\S 2.2$ but to our knowledge, there is no derivation of reduced complexity models in the literature that gets rid of both of them at the same time. This is the main objective of this work.

The shape of water waves results from the competition between nonlinear effects (that make waves steepen) and dispersion effects (that tend to stabilise). The perfect balance between the two corresponds to solitary waves. Waves thus evolve in a given regime corresponding to different orders of magnitude for parameters $\mu$ and $\varepsilon$ that respectively characterise (frequency) dispersion and amplitude dispersion (nonlinearity). These regimes depend mainly on spatial scales: the shallowness parameter $\mu$ describes the ratio of the water depth to the wavelength while $\varepsilon$ is the ratio of the wave amplitude to the water depth. Hence, there seems to have no ultimate model capable of modelling flows from deep water to nearshore areas and at the same time amenable from the computational point of view. Models are thus simplified to match specific spatial scales. The range of regimes then induced in the literature a hierarchy of models derived by means of asymptotic expansions with respect to $\mu$ and $\varepsilon$. We mention a few examples below but readers may refer e.g. to $[35,36]$ for a more exhaustive review of nonlinear/dispersive models for water flows.

In order to model stratified flows i.e. flows where the horizontal velocity significantly differs from its vertical mean and can be hardly approximated by the latter quantity, two approaches can be considered. In the shear model [47,48], a new variable is introduced and may be interpreted as the vertical vorticity. A version with dispersive effects can also be obtained [16]. In the other strategy, the vertical averaging is performed over the whole water column but between intermediate interfaces. This approach is generally referred to as multilayer models. Several versions were introduced depending on the definition of the interfaces. The main advantage of this strategy is to allow any shape of vertical profile of the horizontal velocity over the water column. In the current paper, we proceed similarly to [5] where a multilayer model with mass exchange was derived. This seems to be a robust method. From now on, we refer to the multilayer model with mass exchange as the layerwise dicretisation in order to differ from other multilayer models.

The main objective of this work is to properly derive a reduced complexity model for free surface flows which circumvents the main assumptions usually imposed in geophysics modelling. Different approximations for the hydrodynamic ${ }^{1}$ pressure can be contemplated which leads to a hierarchy of models obtained by means of a layerwise discretisation (LDNH in the sequel). More precisely, three models are introduced corresponding to different degrees of Galerkin approximation. The $\mathrm{LDNH}_{0}$ model is characterised by a layerwise affine approximation for the hydrodynamic pressure and the kinetic energy while the $\mathrm{LDNH}_{2}$ is based on layerwise quadratic approximations for the latter variables. The $\mathrm{LDNH}_{1}$ model is an intermediate system mixing both approaches and taking advantage of homogeneous vertical grids.

The optimal choice between complexity and accuracy is not clear at this stage. Deeper analyses together with numerical experiments are required to confront them but it will be the aim of further works. Nevertheless, it is proven that each model derived in this paper satisfies an exact energy balance. An explicit linear dispersion relation is also provided for each one no matter what the number of layers. In addition we prove that for the three proposed models, the corresponding celerity converges to the exact one for the Euler equations in Airy's theory when the number of layers tends to infinity.

The models derived in this work have the form of a system of conservation laws with source terms coupled with divergence free constraints. Compared to the Euler system, the proposed models allow the easy handling of the free surface since it does not require moving meshes. Moreover, the structure of the models allows to take advantage of robust and accurate numerical techniques developed extensively in the literature for conservation laws. Even if this aspect is not addressed in this paper, the proposed models are well suited for numerical treatments.

\footnotetext{
${ }^{1}$ In the sequel, the non-hydrostatic component of the pressure will be referred to as the hydrodynamic pressure.
} 
The paper is organised as follows: in $\S 2$ we recall the parent model we aim at approximating, namely the free-surface Euler equations. In particular, we describe the state of art of reduced complexity models. Then the models are formulated in $\S 3$. The main advantage of this hierarchy of models lies into the degrees of freedom per layer. A preliminary analysis, including properties such as energy estimates, hydrodynamic balances and linear dispersion relations, is carried out in $\S 4$. We finally specify in $\S 5$ the link between all models to legitimate the notion of hierarchy.

\section{State of art for incompressible free surface flows}

\section{$2.1 \quad$ Euler model}

To model a free surface flow with constant density, we consider the 2D $(x, z)$ Euler system which reads

$$
\left\{\begin{array}{l}
\partial_{x} \underline{u}+\partial_{z} \underline{w}=0 \\
\partial_{t} \underline{u}+\partial_{x}\left(\underline{u}^{2}+\underline{p}\right)+\partial_{z}(\underline{u w})=0, \\
\partial_{t} \underline{w}+\partial_{x}(\underline{u w})+\partial_{z}\left(\underline{w}^{2}+\underline{p}\right)=-g .
\end{array}\right.
$$

The equations are set in a moving domain ${ }^{2}$

$$
\Omega(t)=\left\{(x, z) \in \mathbb{R}^{2} \mid z_{b}(t, x) \leq z \leq \underline{\eta}(t, x)\right\}
$$

where $g>0$ is the gravity acceleration and $\underline{\eta}$ is the unknown water elevation. $z_{b}$ is the topography whose evolution is prescribed by data or another model (erosion, ....). The simplest case corresponds to a topography which does not depend on time but for the sake of generality, we keep both dependencies in the sequel. $\underline{H}=\underline{\eta}-z_{b}$ is then the water height. In the sequel, underlined variables refer to the solutions to the Euler system while bare variables denote numerical solutions. In System (2.1), the unknowns are the velocity field $\underline{\boldsymbol{u}}=(\underline{u}, \underline{w})$ and the pressure $\underline{p}$ in addition to the water elevation $\underline{\eta}$.

The model is supplemented with boundary conditions at the free surface $z=\underline{\eta}(t, x)$

$$
\begin{aligned}
\partial_{t} \underline{\eta}(t, x)+\underline{u}(t, x, \underline{\eta}(t, x)) \partial_{x} \underline{\eta}(t, x)-\underline{w}(t, x, \underline{\eta}(t, x)) & =0, \\
\underline{p}(t, x, \underline{\eta}(t, x)) & =p^{a t m}(t, x),
\end{aligned}
$$

for a given atmospheric pressure $p^{a t m}$. The kinematic condition (2.2a) expresses the fact that no fluid particle crosses the free surface. At the bottom of the domain we impose the no-penetration boundary condition

$$
\partial_{t} z_{b}(t, x)+\underline{u}\left(t, x, z_{b}(t, x)\right) \partial_{x} z_{b}(t, x)-\underline{w}\left(t, x, z_{b}(t, x)\right)=0 .
$$

We consider well-prepared initial conditions insofar as the steady equation in (2.1) must hold initially:

$$
\left\{\begin{array}{l}
\underline{\boldsymbol{u}}(t=0, \cdot)=\boldsymbol{u}^{0}, \quad \text { with } \partial_{x} u^{0}+\partial_{z} w^{0}=0, \\
\underline{p}(t=0, \cdot)=p^{0} .
\end{array}\right.
$$

As we focus on the influence of the non-hydrostatic component of the pressure field, we introduce the hydrodynamic pressure $\underline{q}$ as in [17] defined by

$$
\underline{p}(t, x, z)=p^{a t m}(t, x)+g(\underline{\eta}(t, x)-z)+\underline{q}(t, x, z),
$$

so that System (2.1) also reads

$$
\left\{\begin{array}{l}
\partial_{x} \underline{u}+\partial_{z} \underline{w}=0, \\
\partial_{t} \underline{u}+\partial_{x}\left(\underline{u}^{2}+\underline{q}\right)+\partial_{z}(\underline{u w})=-\partial_{x}\left(g \underline{\eta}+p^{a t m}\right), \\
\partial_{t} \underline{w}+\partial_{x}(\underline{u w})+\partial_{z}\left(\underline{w}^{2}+\underline{q}\right)=0 .
\end{array}\right.
$$

\footnotetext{
${ }^{2}$ It can also be set in a fixed domain $\mathbb{R}^{2} \times\left[0, L_{z}\right]$ with $0<z_{b} \leq \eta<L_{z}$ introducing a color function $\phi$ such that $\phi(t, x, z)=1$ if $z \in\left[z_{b}(t, x), \eta(t, x)\right]$ and $\phi(t, x, z)=0$ otherwise. This function is transported by the flow.
} 
Given this focus, other phenomena such as erosion, surface tension or wind effects are not taken into account.

We underline that even if all the statements are given for the 2D Euler equations (which results in a 1D averaged model), they extend directly to the 3D Euler equations.

\subsection{Reduced complexity models in the literature}

We recall that $\mu$ and $\epsilon$ are respectively the dispersion and nonlinearity parameters.

\subsubsection{Dispersive models}

The first element of the hierarchy is the nonlinear shallow water equations (NLSW) which correspond to the asymptotics $\mu \rightarrow 0$ without regard to $\epsilon$. This non-dispersive model (like weakly dispersive others) benefits from the reduction of space dimension: the low $\mu$-regime - which transcribes the fact that the water depth is negligible compared to horizontal characteristic lengths - comes down to considering a homogeneous horizontal velocity along the vertical axis so that vertical unknowns are left apart. Under this assumption, 3D problems (resp. 2D) are then approximated by 2D models (resp. 1D) which has a strong impact upon the computational time: this legitimates the major interest of the hydrodynamics community for this model.

The NLSW equations were introduced for one space dimension by Saint-Venant [22] and derived more recently in [27] including viscous effects. Two dimensional extensions of NLSW equations with viscosity terms were proposed by Ferrari and Saleri [26] and Marche [41]. Bouchut and Westdickenberg [11] proposed a NLSW-type model valid for an arbitrary topography. Although extensively studied from the numerical point of view (see for instance $[4,30,45,52]$ and references therein) and implemented in industrial codes (HEC-RAS [34], MIKE [21], Telemac [32], Iber [20], ...), the NLSW equations turn out to be irrelevant for some real applications in particular for an arbitrary topography (especially with large variations), when considering stratified flows or when wave breaking is about to occur. These restrictions are mainly due to the fact that $\mu=0$ means the system is non-dispersive or equivalently the pressure field is hydrostatic.

To account for those dispersive effects, a common strategy consists in performing an asymptotic expansion with respect to $\mu$ and/or to $\epsilon$ into the nondimensionalised version of the Euler equations. Resulting models can somehow be considered perturbations of the NLSW equations. When smallness assumptions upon $\mu$ are imposed, such weakly dispersive models are generally called Boussinesq-type models (BTM) after Boussinesq pioneering works [13]. It comprises weakly nonlinear models like [44] when $\epsilon$ is also assumed to be small (together with $\epsilon=\mathcal{O}\left(\mu^{2}\right)$ ) and fully nonlinear models when the expansion is carried out without regard to $\epsilon$ (see [50] in dimension 1 and [29] in dimension 2 with topography). More recently, a large amount of papers were dedicated to the derivation of dispersive models with different orders of accuracy (e.g. $[8,9,35,37,53,54])$ but mainly under the irrotational hypothesis (potential flows). Compared to the NLSW equations, BTM models involve higher order derivatives which complicates numerical treatments $[10,19]$.

To assess these models, one usually relies on the linear wave theory. Indeed, the weakly dispersive assumption is related to the shallow water property and it is crucial to determine the range of applications of models at stake. The associated dispersion relation is derived and compared to the Airy theory (or $1^{\text {st }}$-order Stokes theory) which enables to determine bounds of validity in terms of parameter $k H_{0}$ (where $k$ is the wavenumber and $H_{0}$ the constant water height in which the wave propagates) so that some models are relevant rather in shallow water conditions or up to intermediate water. Comparisons helped modify the original system to improve the match with the Airy's formula; see [39,40,43] for such procedures. While Peregrine [44] used the vertical average of the horizontal velocity, Nwogu [43] based his work on a horizontal velocity at an arbitrary altitude which is left as a degree of freedom in the optimisation process of the dispersion relation. However we must underline three facts to bear in mind: first, models are usually used beyond their domain of validity [38]; second, the "exact formula" used as a reference (Airy) has its own limitations; third, numerical schemes dedicated to dispersive models must be used with mesh sizes which are small enough to correctly catch dispersive effects [33]. 
There exists another procedure to derive models for fluid flows. Based on the same procedure as the NLSW equations, non-hydrostatic models $(\mathrm{NH})$ were introduced $[15,17,18,51,55]$ by means of averaging processes over the water depth. Unlike BTM models where high order derivatives are involved, NH systems have additional unknowns and equations with $1^{\text {st }}$ order derivatives, namely the non-hydrostatic pressure (also called hydrodynamic pressure [17]) and the evolution equation for the vertical velocity. In addition to the reduction of differential orders, boundary conditions may be simpler to deal with. Such systems were for instance applied to the modelling of the swash zone [56]. The numerical algorithm is split into two steps: on the one hand, a standard resolution of the hydrostatic (hyperbolic) part and on the other hand, a projection method to solve the elliptic equation for the dispersive part.

\subsubsection{Multilayer models}

Several models attempt to better reproduce the vertical profile of the horizontal velocity. This approach is based on a splitting of the water column into an arbitrary number of layers which can be interpreted as a semi-discretisation along the vertical axis. In addition, this allows to deal with stratified fluids. Several definitions of the layers can be considered.

First attempts $[17,42]$ relied on (horizontal) layers of fixed thicknesses. In these cases, the free surface motion implied that the fluid domain does not coincide with the grid: some control volumes are then partially filled by the fluid. A similar problem occurs for control volumes crossing the bathymetry and which are also partially filled. Alternatively the bathymetry can be approximated by the lowest layer of the volume. To circumvent this issue, another possibility consists in considering the well-known $\sigma$-coordinates [46]. Nevertheless, $\sigma$ coordinates present some drawbacks mainly in areas with high gradients of the bathymetry and in wet/dry fronts. Let us remark that an interpretation of $\sigma$-coordinates as an ALE formulation has been proposed in [23], where authors also introduced an improvement of the $\sigma$-coordinates transformation.

Another technique was introduced in [3] under the assumption of hydrostatic pressures. The author introduced a piecewise vertical integration of Euler equations by assuming a specific vertical profile of the velocity field inside each layer (a constant profile for the horizontal velocity and a linear profile of the vertical component). The thickness of the layers is proportional to the total height of the fluid, which is equivalent to considering a moving grid varying in space and time. The multilayer method has been improved in [5] with the incorporation of mass transfer terms between layers. Our approach is a improvement of this work to take into account the dispersive effect. The overall technique can be interpreted as a splitting technique where the vertical discretisation is performed by means of a discontinuous approach. From this point of view, the transfer terms are related to the jump conditions across interfaces that defined the "vertical" boundaries of layers [24].

This vertical discretisation was also considered in several papers in the literature $[6,38,49]$ for the case of hydrodynamic pressure. In [49] the author adapted the technique proposed in [5] to the non-hydrostatic case. The corresponding derivation amounts to supposing within each layer a constant vertical profile of both horizontal and vertical components of the velocity fields, a parabolic profile for the pressure and a linear vertical velocity for the integration of the incompressibility condition. In [6] the authors proposed a vertical integration that corresponds to a constant profile of the horizontal velocity, a linear profile of the vertical velocity and a linear profile of the pressure. The common hypothesis in these references is thus that the horizontal velocity has a constant vertical profile in each layer and is discontinuous at the interfaces. A different assumption holds in [38] where, under the irrotational hypothesis, a multilayer model is derived by using a global continuous profile of the horizontal velocity which is quadratic within each layer. Nevertheless to our knowledge, aforementioned models do not satisfy an exact energy balance which is an important feature in addition to linear wave properties. 


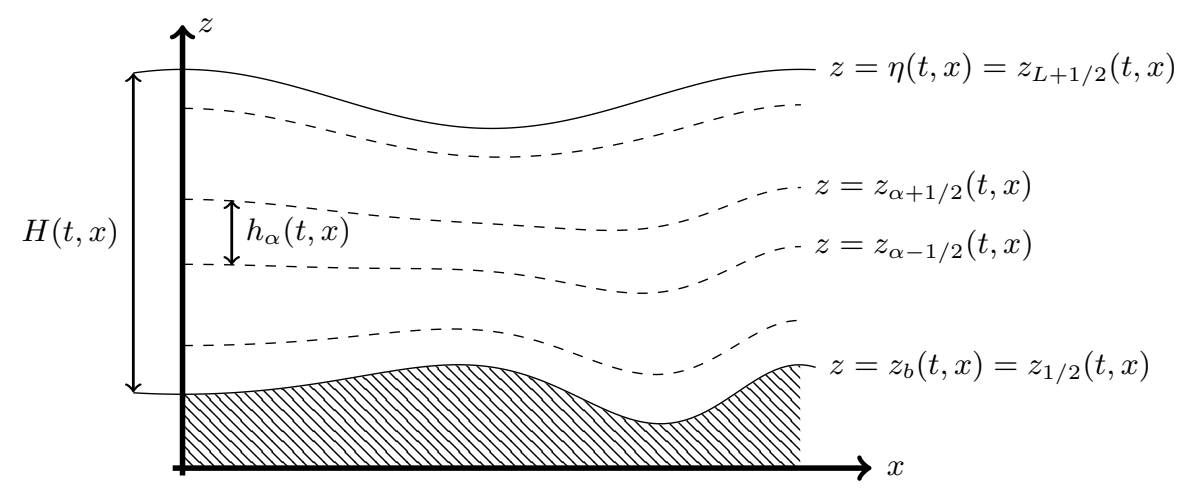

Figure 1: Interpretation of the layerwise discretisation in the vertical plan.

\section{Description of the hierarchy of models}

Before giving the formulation of the three models comprising the hierarchy, we first introduce the multilayer framework as well as the approximation procedure we shall consider in the derivation.

\subsection{Setting of the multilayer framework and notations}

Given a positive water height $H(t, x)$, a number of layers $L \geq 1$ and a convex combination $\left(\ell_{\alpha}\right)_{1 \leq \alpha \leq L}$ such that

$$
\ell_{\alpha} \in(0,1], \sum_{\alpha=1}^{L} \ell_{\alpha}=1
$$

we set

$$
h_{\alpha}(t, x)=\ell_{\alpha} H(t, x) \Longrightarrow H=\sum_{\alpha=1}^{L} h_{\alpha} .
$$

As depicted on Figure 1, the flow is split along the vertical axis into $L \geq 1$ layers denoted by

$$
\mathcal{L}_{\alpha}(t, x)=\left[z_{\alpha-1 / 2}(t, x), z_{\alpha+1 / 2}(t, x)\right] \quad \text { for } \quad \alpha \in\{1, \ldots, L\}, \quad \text { where } \quad z_{\alpha+1 / 2}=z_{b}+\sum_{\beta=1}^{\alpha} h_{\beta} .
$$

So that $z_{b}=z_{1 / 2}<z_{3 / 2}<\ldots<z_{L+1 / 2}=\eta=z_{b}+H$, and $h_{\alpha}(t, x)=z_{\alpha+1 / 2}(t, x)-z_{\alpha-1 / 2}(t, x)$.

Layers are not physically based except when focusing on immiscible layers. For the sake of clarity, middle points of layer $\mathcal{L}_{\alpha}$ are denoted by

$$
z_{\alpha}=\frac{z_{\alpha+1 / 2}+z_{\alpha-1 / 2}}{2} .
$$

We shall note in the sequel the mean value of a function $(t, x, z) \mapsto f(t, x, z)$ over $\mathcal{L}_{\alpha}$

$$
\langle f\rangle_{\alpha}(t, x)=\frac{1}{h_{\alpha}(t, x)} \int_{z_{\alpha-1 / 2}(t, x)}^{z_{\alpha+1 / 2}(t, x)} f(t, x, z) \mathrm{d} z .
$$

As we describe bellow the unknowns of the multilayer approach may be discontinuous across layer interfaces. Then, for a general function $f$, we introduce the classical notations

$$
f_{\alpha+1 / 2}^{+}(t, x)=\lim _{\substack{z \rightarrow z_{\alpha+1 / 2}(t, x) \\ z>z_{\alpha+1 / 2}(t, x)}} f_{\mid \mathcal{L}_{\alpha+1}(t, x)}(t, x, z), \quad f_{\alpha+1 / 2}^{-}(t, x)=\lim _{\substack{z \rightarrow z_{\alpha+1 / 2}(t, x) \\ z<z_{\alpha+1 / 2}(t, x)}} f_{\mid \mathcal{L}_{\alpha}(t, x)}(t, x, z) .
$$

As a consequence, jumps across $z=z_{\alpha+1 / 2}$ correspond to

$$
\llbracket f \rrbracket_{\alpha+1 / 2}=f_{\alpha+1 / 2}^{+}-f_{\alpha+1 / 2}^{-} .
$$


Let us introduce a normal vector to the interface $z=z_{\alpha+1 / 2}(t, x)$ which points upward and defined by

$$
\boldsymbol{n}_{\alpha+1 / 2}(t, x)=\left(\begin{array}{c}
-\partial_{x} z_{\alpha+1 / 2}(t, x) \\
1
\end{array}\right) .
$$

As in [24], we assume that both the normal velocity and the pressure effects are continuous across the layer interfaces, i.e.

$$
\begin{aligned}
\llbracket \underline{\boldsymbol{u}} \rrbracket_{\alpha+1 / 2} \cdot \boldsymbol{n}_{\alpha+1 / 2} & =0, \\
\llbracket \underline{q} \rrbracket_{\alpha+1 / 2} & =0 .
\end{aligned} \quad \text { that is } \llbracket \underline{w} \rrbracket_{\alpha+1 / 2}=\llbracket \underline{u} \rrbracket_{\alpha+1 / 2} \partial_{x} z_{\alpha+1 / 2},
$$

Hypothesis (3.2a) implies that layers remain adjoining and no void appears within the flow. Let us remark that both are somehow related to the divergence free constraint $(2.5 \mathrm{a})$. In fact, the continuity of the normal component of the velocity field naturally arises from the jump condition associated to an isolated discontinuity (see [24]). On the other hand in the incompressible framework, the pressure field may be identified as the Lagrange multiplier associated to (2.5a).

We also denote by

$$
\widetilde{f}_{\alpha+1 / 2}=\left(1-\gamma_{\alpha+1 / 2}\right) f_{\alpha+1 / 2}^{+}+\gamma_{\alpha+1 / 2} f_{\alpha+1 / 2}^{-}, \quad \gamma_{\alpha+1 / 2} \in[0,1],
$$

any convex combination providing a mean value at the interface. The convex coefficient might be different from one unknown to another but we decide to take the same throughout the article. When $f$ is continuous across the interface i.e. $\llbracket f \rrbracket_{\alpha+1 / 2}=0,(3.3)$ reduces to $\widetilde{f}_{\alpha+1 / 2}=f_{\alpha+1 / 2}$.

The main assumption in the multilayer approach consists in considering that $\underline{u}$ is approximated with respect to the vertical variable $z$ by a layerwise constant function:

$$
\underline{u}(t, x, z)=\sum_{\alpha=1}^{L} u_{\alpha}(t, x) \mathbb{1}_{\left\{\mathcal{L}_{\alpha}(t, x)\right\}}(z)+\mathcal{E}_{L},
$$

where $u_{\alpha}$ is an approximation of $\langle\underline{u}\rangle_{\alpha}$ and $\mathcal{E}_{L}$ is the truncation error assumed to be at least of order $L^{-1}$. The classical Saint-Venant model [22] corresponds to $L=1$ which is a coarse mesh with a single vertical cell. Any $L>1$ is thus expected to provide more accurate results.

This decomposition is relevant for small layer thicknesses (see (3.1) for notations) where

$$
\max _{\alpha} \ell_{\alpha}=\varepsilon
$$

It is the case in particular for homogeneous grids when $L=\varepsilon^{-1}$. The asymptotics $L \rightarrow+\infty$ enables to study the consistency between semi-discrete models $\mathrm{LDNH}_{\nu}$ and the Euler system (2.5).

If we assume that

$$
\underline{u}(t, x, z)=\langle\underline{u}\rangle_{\alpha}(t, x)+\varepsilon \underline{u}_{\alpha}^{\prime}(t, x, z), \quad z \in \mathcal{L}_{\alpha}(t, x),
$$

with $\left\langle\underline{u}_{\alpha}^{\prime}\right\rangle_{\alpha}=0$ and $\underline{u}_{\alpha}^{\prime}=\mathcal{O}_{\varepsilon}(1)$, then the integration of Eq. (2.5a) over $\left[\underline{z}_{\alpha-1 / 2}, z\right]$ yields

$$
\underline{w}_{\mid \mathcal{L}_{\alpha}(t, x)}(t, x, z)=\underline{w}_{\alpha-1 / 2}^{+}(t, x)-\left(z-\underline{z}_{\alpha-1 / 2}(t, x)\right) \partial_{x}\langle\underline{u}\rangle_{\alpha}(t, x)-\varepsilon \int_{\underline{z}_{\alpha+1 / 2}}^{z} \partial_{x} \underline{u}_{\alpha}^{\prime}(t, x, \zeta) \mathrm{d} \zeta .
$$

In particular this implies

$$
\langle\underline{w}\rangle_{\alpha}=\underline{w}_{\alpha-1 / 2}^{+}-\frac{h_{\alpha}}{2} \partial_{x}\langle\underline{u}\rangle_{\alpha}-\varepsilon \int_{z_{\alpha-1 / 2}}^{z_{\alpha+1 / 2}} \frac{z_{\alpha+1 / 2}-z}{h_{\alpha}} \partial_{x} \underline{u}^{\prime}(\cdot, \cdot, z) \mathrm{d} z .
$$

Hence the combination of the two last equalities yields

$$
\underline{w}_{\mid \mathcal{L}_{\alpha}(t, x)}(t, x, z)=\langle\underline{w}\rangle_{\alpha}(t, x)-\left(z-z_{\alpha}(t, x)\right) \partial_{x}\langle\underline{u}\rangle_{\alpha}(t, x)+\mathcal{O}(\varepsilon) .
$$


Consequently, the vertical component $\underline{w}$ of the velocity field may be approximated by a layerwise affine discontinuous function, i.e.

$$
\underline{w}(t, x, z)=\sum_{\alpha=1}^{L}\left[w_{\alpha}(t, x)-\left(z-z_{\alpha}(t, x)\right) \partial_{x} u_{\alpha}(t, x)\right] \mathbb{1}_{\left\{\mathcal{L}_{\alpha}(t, x)\right\}}(z)+\mathcal{O}(\varepsilon)
$$

which allows for discontinuities across layer interfaces $z=z_{\alpha+1 / 2}(t, x)$. This is incorporated in $\mathrm{LDNH}_{2}$ $(\S 3.2)$ and $\mathrm{LDNH}_{1}(\S 3.3)$. But an alternative to (3.7) consists in approximating $\underline{w}$ by a layerwise constant function, namely

$$
\underline{w}(t, x, z)=\sum_{\alpha=1}^{L} w_{\alpha}(t, x) \mathbb{1}_{\left\{\mathcal{L}_{\alpha}(t, x)\right\}}(z)+\mathcal{O}(\varepsilon) .
$$

This choice leads to Model $\mathrm{LDNH}_{0}(\S 3.4)$.

For the hydrodynamic counterpart pressure field $\underline{q}$, we choose a continuous approximation (see equation $(3.2 \mathrm{~b}))$. The degree of the approximation polynomial is then prescribed by Eq. (2.5c): $\left.q\right|_{\mathcal{L}_{\alpha}(t, x)} \in \mathbb{P}_{2}$ if $\left.w\right|_{\mathcal{L}_{\alpha}(t, x)} \in \mathbb{P}_{1}$ and $\left.q\right|_{\mathcal{L}_{\alpha}(t, x)} \in \mathbb{P}_{1}$ if $\left.w\right|_{\mathcal{L}_{\alpha}(t, x)} \in \mathbb{P}_{0}$

As it will be shown in the sequel, the discretisation procedure carried out in this paper leads to a hierarchy of models $\left(\mathrm{LDNH}_{\nu}\right)_{\nu \in\{0,1,2\}}$ corresponding to different orders of approximation for each unknown. We present in the following subsection the most complete model $\mathrm{LDNH}_{2}$ and then lower-order counterparts $\mathrm{LDNH}_{1}$ and $\mathrm{LDNH}_{0}$. The derivations of these models are detailed respectively in Section $4.1\left(\mathrm{LDNH}_{2}\right)$, Appendices $\mathrm{B}$ $\left(\mathrm{LDNH}_{1}\right)$ and $\mathrm{C}\left(\mathrm{LDNH}_{0}\right)$. The main differences between models will be investigated in Section 5 .

\subsection{Model $\mathrm{LDNH}_{2}$}

The main dispersive multilayer model (3.9)-(3.10) is named $\mathrm{LDNH}_{2}$. This model relies on a multilayer approach based on a discontinuous $\mathbb{P}_{0}$-approximation for $u$, discontinous $\mathbb{P}_{1}$ for $w$ and continuous piecewise $\mathbb{P}_{2}$ for $q$. It reads

$$
\partial_{t} H+\partial_{x}(H \bar{u})=0, \quad \bar{u}=\sum_{\alpha=1}^{L} \ell_{\alpha} u_{\alpha},
$$

and for $\alpha \in\{1, \ldots, L\}$

$$
\begin{aligned}
& \partial_{t}\left(h_{\alpha} u_{\alpha}\right)+\partial_{x}\left(h_{\alpha} u_{\alpha}^{2}+h_{\alpha} q_{\alpha}\right)+ \widetilde{u}_{\alpha+1 / 2} \Gamma_{\alpha+1 / 2}-\partial_{x} z_{\alpha+1 / 2} q_{\alpha+1 / 2} \\
&-\widetilde{u}_{\alpha-1 / 2} \Gamma_{\alpha-1 / 2}+\partial_{x} z_{\alpha-1 / 2} q_{\alpha-1 / 2}=-h_{\alpha} \partial_{x}\left(g \eta+p^{a t m}\right), \\
& \partial_{t}\left(h_{\alpha} w_{\alpha}\right)+\partial_{x}\left(h_{\alpha} u_{\alpha} w_{\alpha}\right)+\widetilde{w}_{\alpha+1 / 2} \Gamma_{\alpha+1 / 2}+q_{\alpha+1 / 2}-\widetilde{w}_{\alpha-1 / 2} \Gamma_{\alpha-1 / 2}-q_{\alpha-1 / 2}=0, \\
& \partial_{t}\left(h_{\alpha} \sigma_{\alpha}\right)+\partial_{x}\left(h_{\alpha} \sigma_{\alpha} u_{\alpha}\right)=2 \sqrt{3}\left[q_{\alpha}-\frac{q_{\alpha+1 / 2}+q_{\alpha-1 / 2}}{2}\right. \\
&\left.-\Gamma_{\alpha+1 / 2}\left(\frac{h_{\alpha} \partial_{x} u_{\alpha}}{12}+\frac{\widetilde{w}_{\alpha+1 / 2}-w_{\alpha}}{2}\right)+\Gamma_{\alpha-1 / 2}\left(\frac{h_{\alpha} \partial_{x} u_{\alpha}}{12}+\frac{w_{\alpha}-\widetilde{w}_{\alpha-1 / 2}}{2}\right)\right],
\end{aligned}
$$

together with some diagnostic equations that transcribe the free divergence constraint (2.5a)

$$
\begin{aligned}
& \partial_{x} u_{\alpha}+\frac{w_{\alpha+1 / 2}^{-}-w_{\alpha}}{h_{\alpha} / 2}=0 \\
& w_{\alpha+1 / 2}^{+}-\partial_{t} z_{b}-u_{\alpha+1} \partial_{x} z_{\alpha+1 / 2}+\sum_{\beta=1}^{\alpha} \partial_{x}\left(h_{\beta} u_{\beta}\right)=0 \\
& \sigma_{\alpha}+\frac{h_{\alpha} \partial_{x} u_{\alpha}}{2 \sqrt{3}}=0 .
\end{aligned}
$$


It is supplemented with discrete jump conditions (3.2a) which become, for $\alpha \in\{1, \ldots, L-1\}$,

$$
w_{\alpha+1 / 2}^{+}-w_{\alpha+1 / 2}^{-}=\partial_{x} z_{\alpha+1 / 2}\left(u_{\alpha+1}-u_{\alpha}\right),
$$

and boundary conditions

$$
\begin{aligned}
q_{L+1 / 2} & =0, \\
w_{L+1 / 2}^{-} & =\partial_{t} \eta+u_{L} \partial_{x} \eta .
\end{aligned}
$$

We mention that initial conditions that must supplement (3.9-3.10) are expected to satisfy diagnostic equations (3.9e-3.9g).

$\Gamma_{\alpha \pm 1 / 2}$ terms involved in (3.9) account for mass transfer ${ }^{3}$ across interfaces and are defined by

$$
\Gamma_{\alpha+1 / 2}=\sum_{\beta=\alpha+1}^{L} \partial_{x}\left(h_{\beta}\left(u_{\beta}-\bar{u}\right)\right) .
$$

The derivation of Equations (3.9-3.10) is described in Section 4.1.

Let us mention that the unknown $\sigma_{\alpha}$ is an approximation of the signed standard deviation $\underline{\sigma}_{\alpha}$, verifying

$$
\underline{\sigma}_{\alpha}^{2}=\left\langle\left(\underline{w}-\langle\underline{w}\rangle_{\alpha}\right)^{2}\right\rangle_{\alpha},
$$

with the same sign as $\partial_{z} \underline{w}$. Taking into account (3.7), we have

$$
\underline{\sigma}_{\alpha}^{2}=\frac{h_{\alpha}^{2}\left(\partial_{x}\langle\underline{u}\rangle_{\alpha}\right)^{2}}{12}+\mathcal{O}(\varepsilon)
$$

that is consistent with equation $(3.9 \mathrm{~g})$ verified by $\sigma_{\alpha}$. Moreover we obtain

$$
\underline{w}_{\alpha+1 / 2}^{-}=\langle\underline{w}\rangle_{\alpha}+\sqrt{3} \underline{\sigma}_{\alpha}+\mathcal{O}(\varepsilon) \quad \text { and } \quad \underline{w}_{\alpha-1 / 2}^{+}=\langle\underline{w}\rangle_{\alpha}-\sqrt{3} \underline{\sigma}_{\alpha}+\mathcal{O}(\varepsilon) .
$$

Remark 1 Eq. (3.9b) also reads

$$
\begin{aligned}
\partial_{t}\left(h_{\alpha} u_{\alpha}\right)+\partial_{x}\left(h_{\alpha} u_{\alpha}^{2}+g \frac{h_{\alpha}^{2}}{2 \ell_{\alpha}}+h_{\alpha} q_{\alpha}\right)+\widetilde{u}_{\alpha+1 / 2} \Gamma_{\alpha+1 / 2}-\partial_{x} z_{\alpha+1 / 2} q_{\alpha+1 / 2} \\
\quad-\widetilde{u}_{\alpha-1 / 2} \Gamma_{\alpha-1 / 2}+\partial_{x} z_{\alpha-1 / 2} q_{\alpha-1 / 2}=-g h_{\alpha} \partial_{x} z_{b}-h_{\alpha} \partial_{x} p^{a t m}
\end{aligned}
$$

or equivalently

$$
\partial_{t}\left(h_{\alpha} u_{\alpha}\right)+\partial_{x}\left(h_{\alpha} u_{\alpha}^{2}+h_{\alpha} p_{\alpha}\right)+\widetilde{u}_{\alpha+1 / 2} \Gamma_{\alpha+1 / 2}-\partial_{x} z_{\alpha+1 / 2} p_{\alpha+1 / 2}-\widetilde{u}_{\alpha-1 / 2} \Gamma_{\alpha-1 / 2}+\partial_{x} z_{\alpha-1 / 2} p_{\alpha-1 / 2}=0
$$

where $p_{\alpha}$ and $p_{\alpha \pm 1 / 2}$ are inferred from (2.4). Hence dispersive terms are not only related to spatial derivatives but also to coupling terms between layers. If we impose the hydrostatic assumption by setting $q_{\alpha}=0$ and $q_{\alpha+1 / 2}=0$, we recover the multilayer model from [5] made only of (3.9a-3.9b) as fewer unknowns are involved. ${ }^{4}$

\footnotetext{
${ }^{3}$ In some works (see for instance $[3,12]$ ), transfer terms are set to 0 in particular when layers correspond to non-miscible fluids. In many articles [5,24], the mass flux is denoted by $\mathcal{G}_{\alpha+1 / 2}$ and is such that $\mathcal{G}_{\alpha+1 / 2}=-\Gamma_{\alpha+1 / 2}$. In [57], the mass transfer term $\Gamma_{\alpha+1 / 2}$ is denoted by $\omega_{\alpha+1 / 2}$ and in [6] by $\Delta w_{\alpha+1 / 2}$.

${ }^{4}$ The vertical component of the velocity field is computed a posteriori using (3.9f).
} 
Multilayer Serre-Green-Naghdi model. We can see $\mathrm{LDNH}_{2}$ model as a generalisation of the SerreGreen-Naghdi model. Firstly, because when a single layer is considered $(L=1)$, the $\mathrm{LDNH}_{2}$ model reduces to the so-called Serre-Green-Naghdi model [29,37,50] (also referred to as nonlinear Boussinesq equations [53]). The proof consists of simple calculations: for the sake of simplicity, let us consider that $p^{a t m}=0$ and $\partial_{t} z_{b}=0$. Indices $b$ and $s$ refer to bottom and surface data. For $L=1$, due to $\Gamma_{b}=\Gamma_{s}=0$, Eqs. (3.9)-(3.10) read

$$
\left\{\begin{array}{l}
\partial_{t} H+\partial_{x}(H u)=0, \\
H\left(\partial_{t} u+u \partial_{x} u\right)+\partial_{x}(H q)=-q_{b} \partial_{x} z_{b}-g H \partial_{x} \eta \\
q_{b}=H\left(\partial_{t} w+u \partial_{x} w\right), \\
q=\frac{q_{b}}{2}-\frac{H}{12}\left[\partial_{t}\left(H \partial_{x} u\right)+u \partial_{x}\left(H \partial_{x} u\right)\right], \\
w=w_{s}+\frac{H}{2} \partial_{x} u, \\
w_{s}=u \partial_{x} z_{b}-H \partial_{x} u .
\end{array}\right.
$$

Straightforward calculations show that this system is equivalent to

$$
\left\{\begin{array}{l}
\partial_{t} \eta+\partial_{x}(H u)=0 \\
H\left(\partial_{t} u+u \partial_{x} u+g \partial_{x} \eta\right)+\partial_{x}\left(H^{2}\left(\frac{\mathcal{P}}{3}+\frac{\mathcal{Q}}{2}\right)\right)+H \partial_{x} z_{b}\left(\frac{\mathcal{P}}{2}+\mathcal{Q}\right)=0 \\
\mathcal{P}=-H\left(\partial_{t x}^{2} u+u \partial_{x x}^{2} u-\left(\partial_{x} u\right)^{2}\right) \\
\mathcal{Q}=\partial_{x} z_{b}\left(\partial_{t} u+u \partial_{x} u\right)+u^{2} \partial_{x x}^{2} z_{b} .
\end{array}\right.
$$

We recover the Serre-Green-Naghdi model under the form of [19].

More generally, this dispersive model with arbitrary number of layers can be expressed as a Boussinesq type model with high order derivatives. For instance, the standard deviation $\sigma$ characterised by $(3.9 \mathrm{~g})-$ used like in mixed formulations for finite-element methods - could have been incorporated in (3.9d) which would have resulted in second order derivatives for $u$. Similarly, the formulation (3.9)-(3.10) can be expressed with a lower number of unknowns by inserting (3.9e-3.9g) into (3.9b-3.9d). Then $q_{\alpha}$ can be extracted from $(3.9 \mathrm{~d})$ and inserted into (3.9b).

Third of all, it is well known that if we assume that $u_{\alpha}=\bar{u}$ for all $\alpha \in\{1, \ldots, L\}$ in the inviscid multilayer shallow water model presented in [5], we recover the classical shallow water model. In what follows we prove that under this assumption $\mathrm{LDNH}_{2}$ models recover the Serre-Green-Naghdi model.

If we assume that $u_{\alpha}=\bar{u}$ for all $\alpha \in\{1, \ldots, L\}$ by (3.11), we directly obtain $\Gamma_{\alpha+1 / 2}=0$. Then summing (3.9b) and (3.9c) over $\alpha$ and using the same definition for $\bar{w}$ and $\bar{q}$ as in (3.9a) for $u$, we obtain

$$
\left\{\begin{array}{l}
\partial_{t}(H \bar{u})+\partial_{x}\left(H\left(\bar{u}^{2}+\bar{q}\right)\right)+q_{b} \partial_{x} z_{b}=-H \partial_{x}\left(g \eta+p^{a t m}\right), \\
\partial_{t}(H \bar{w})+\partial_{x}(H \bar{u} \bar{w})-q_{b}=0
\end{array}\right.
$$

Then (3.13b) and (3.13c) are recovered. Due to $u_{\alpha+1}=u_{\alpha}$, (3.10a) yields $\widetilde{w}_{\alpha+1 / 2}=w_{\alpha+1 / 2}^{ \pm}=\partial_{t} z_{b}+$ $\bar{u} \partial_{x} z_{\alpha+1 / 2}-\sum_{1 \leq \beta \leq \alpha} \ell_{\beta} \partial_{x}(H \bar{u})$. We deduce that $\widetilde{w}_{\alpha+1 / 2}=\widetilde{w}_{\alpha-1 / 2}-\ell_{\alpha} H \partial_{x} \bar{u}$ which, together with (3.9e), shows that all points $\left(z_{\alpha+1 / 2}, w_{\alpha+1 / 2}\right)$ belong to the same straight line. Therefore the linear function $\hat{w}(t, x, z)=w_{s}(t, x)+(\eta(t, x)-z) \partial_{x} \bar{u}(t, x)$ satisfies $\hat{w}_{\mid z_{\alpha+1 / 2}}=w_{\alpha+1 / 2}$. Moreover $\langle\hat{w}\rangle_{\alpha}=w_{\alpha}$. Hence (3.13e) and (3.13f) also hold. We deduce that (3.9c) reads

$$
q_{\alpha+1 / 2}-q_{\alpha-1 / 2}=-\ell_{\alpha} H\left(\partial_{t}\left(\bar{u} \partial_{x} z_{b}\right)+\bar{u} \partial_{x}\left(\bar{u} \partial_{x} z_{b}\right)\right)-\ell_{\alpha} \Lambda_{\alpha} H\left(\partial_{t}\left(H \partial_{x} \bar{u}\right)+\bar{u} \partial_{x}\left(H \partial_{x} \bar{u}\right)\right)
$$

with $\Lambda_{\alpha}=\frac{\ell_{\alpha}}{2}-\sum_{\beta=1}^{\alpha} \ell_{\beta}$. Let us introduce the polynomial $\hat{q}$

$$
\hat{q}(t, x, z)=(\eta(t, x)-z)^{2} k_{2}(t, x)+(\eta(t, x)-z) k_{1}(t, x),
$$




$$
\begin{aligned}
& k_{2}(t, x)=\frac{1}{2 H(t, x)}\left(\partial_{t}\left(H \partial_{x} \bar{u}\right)+\bar{u} \partial_{x}\left(H \partial_{x} \bar{u}\right)\right), \\
& k_{1}(t, x)=\partial_{t}\left(\bar{u} \partial_{x} z_{b}\right)+\bar{u} \partial_{x}\left(\bar{u} \partial_{x} z_{b}\right)-2 H(t, x) k_{2}(t, x) .
\end{aligned}
$$

We verify that $\hat{q}\left(t, x, z_{\alpha+1 / 2}(t, x)\right)=q_{\alpha+1 / 2}(t, x)$. In particular $\hat{q}\left(t, x, z_{b}(x)\right)=q_{b}(t, x)$.

As for any 2nd-order polynomial, the following property holds for $\hat{q}$

$$
\frac{1}{b-a} \int_{a}^{b} \hat{q}(t, x, z) \mathrm{d} z-\frac{\hat{q}(t, x, b)+\hat{q}(t, x, a)}{2}=-k_{2}(t, x) \frac{(b-a)^{2}}{6} .
$$

Using (3.9d), the latter equality leads to $\langle\hat{q}\rangle_{\alpha}=q_{\alpha}$ and finally to (3.13d).

\subsection{Model $\mathrm{LDNH}_{1}$}

This case is restricted to homogeneous tessellations of intervals $\left[z_{b}(t, x), H(t, x)\right]$, i.e.

$$
\forall \alpha \in\{1, \ldots, L\}, \ell_{\alpha}=\frac{1}{L} \text { with } L \gg 1 .
$$

Truncating quadratic terms in the approximation process leads to an intermediate model, named $\mathrm{LDNH}_{1}$, and consists of

- The mass conservation law (3.9a);

- The momentum conservation equations (3.9b-3.9c);

- Eq. (3.9d) is replaced by

$$
\begin{aligned}
q_{\alpha}=\frac{q_{\alpha+1 / 2}+q_{\alpha-1 / 2}}{2}+\Gamma_{\alpha+1 / 2}\left(\frac{H \widetilde{4 L} \widetilde{\left(\partial_{x} u\right)_{\alpha+1 / 2}}}{}+\frac{\left.\widetilde{w}_{\alpha+1 / 2}-w_{\alpha}\right)}{2}\right) \\
-\Gamma_{\alpha-1 / 2}\left(\frac{H}{4 L} \widetilde{\left(\partial_{x} u\right)_{\alpha-1 / 2}}+\frac{w_{\alpha}-\widetilde{w}_{\alpha-1 / 2}}{2}\right)
\end{aligned}
$$

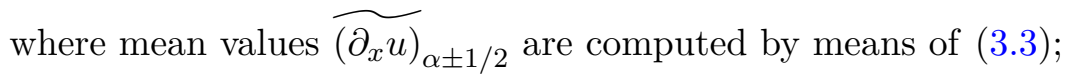

- Divergence constraints (3.9e-3.9f-3.9g);

- Jump conditions (3.10a) and boundary conditions (3.10b-3.10c)

Main unknowns of the resulting system are $\left(H, u_{\alpha}, w_{\alpha}, q_{\alpha+1 / 2}\right)$ as other unknowns are directly computed from algebraic relations (3.9e), (3.9f), (3.9g) and (3.14). This corresponds to a staggered grid where velocity and pressure fields are located at different places in the same spirit as in the MAC method [31].

The derivation of this model is detailed in Section B.

Link with single-layer models. Similarly to the $\mathrm{LDNH}_{2}$ model, the $\mathrm{LDNH}_{1}$ system is related to another one studied in the literature as stated in the following remark. When a single layer is considered $(L=1)$, the $\mathrm{LDNH}_{1}$ model reduces to the dispersive depth-averaged model studied in $[1,2,15]$. See also [55] for a similar model.

To prove it, we keep notations from the previous section. For $L=1$, the $\mathrm{LDNH}_{1}$ model reads

$$
\left\{\begin{array}{l}
\partial_{t} H+\partial_{x}(H u)=0 \\
\partial_{t}(H u)+\partial_{x}\left(H u^{2}+H q\right)=-q_{b} \partial_{x} z_{b}-g H \partial_{x} \eta \\
\partial_{t}(H w)+\partial_{x}(H u w)=q_{b} \\
q=\frac{q_{b}}{2} \\
w=w_{s}+\frac{H}{2} \partial_{x} u \\
w_{s}=u \partial_{x} z_{b}-H \partial_{x} u
\end{array}\right.
$$


We then recover the aforementioned model

$$
\left\{\begin{array}{l}
\partial_{t} \eta+\partial_{x}(H u)=0 \\
\partial_{t}(H u)+\partial_{x}\left(H u^{2}+g \frac{H^{2}}{2}+H q\right)=-(g H+2 q) \partial_{x} z_{b} \\
\partial_{t}(H w)+\partial_{x}(H u w)=2 q \\
\partial_{x}(H u)-u \partial_{x}\left(H+2 z_{b}\right)+2 w=0
\end{array}\right.
$$

\subsection{Model $\mathrm{LDNH}_{0}$}

The simplest model named $\mathrm{LDNH}_{0}$ corresponds to a $\mathbb{P}_{0}$-approximation for $u$ and $w$ and $\mathbb{P}_{1}$ for $q$.

The model consists of

- The mass conservation law (3.9a);

- The momentum conservation equations (3.9b-3.9c) but we underline that the mean value $\widetilde{w}_{\alpha+1 / 2}$ used in $(3.9 \mathrm{c})$ reduces to

$$
\widetilde{w}_{\alpha+1 / 2}=\gamma_{\alpha+1 / 2} w_{\alpha}+\left(1-\gamma_{\alpha+1 / 2}\right) w_{\alpha+1}
$$

as $w$ is layerwise constant in $\mathrm{LDNH}_{0}$;

- Divergence constraints (3.9f) and (3.9d) are substituted by

$$
\begin{gathered}
w_{\alpha}-\partial_{t} z_{b}-u_{\alpha} \partial_{x} z_{\alpha}+\sum_{\beta=1}^{\alpha-1} \partial_{x}\left(h_{\beta} u_{\beta}\right)+\frac{1}{2} \partial_{x}\left(h_{\alpha} u_{\alpha}\right)=0, \\
q_{\alpha}=\frac{q_{\alpha+1 / 2}+q_{\alpha-1 / 2}}{2},
\end{gathered}
$$

- Boundary condition (3.10b).

The derivation of the $\mathrm{LDNH}_{0}$ model is given in Section C.

Link with single-layer models. When a single layer is considered $(L=1)$, the $\mathrm{LDNH}_{0}$ model coincides with the $\mathrm{LDNH}_{1}$ model and thus also reduces to the dispersive depth-averaged model studied in $[1,2,15]$.

\section{Analysis of the $\mathrm{LDNH}_{2}$-model}

The models stated in Section 3 are analysed in the sequel. We focus on the $\mathrm{LDNH}_{2}$-model and transfer the analysis of other models in the appendix. However we specify when results hold for the three of them and refer to specific appendices when it is not the case. In particular, we prove that $\mathrm{LDNH}_{2}$ satisfies an energy inequality. We also provide an explicit linear dispersion relation no matter what the number of layers $L$ which turns out to converge towards Airy's formula when $L \rightarrow+\infty$.

\subsection{Derivation}

\subsubsection{Layer averaging}

Let us propose a general way to discretise in a single direction conservative equations with source terms. The toy model is

$$
\partial_{t} \mathbf{R}+\partial_{x}(u \mathbf{R}+\mathbf{P})+\partial_{z}(w \mathbf{R}+\mathbf{Q})=\mathbf{S}
$$


where $\mathbf{R}, \mathbf{P}, \mathbf{Q}$ and $\mathbf{S}$ take values in $\mathbb{R}^{p}$. If $\bar{X}_{\alpha}$ denotes an approximation of $\langle X\rangle_{\alpha}$, an integration in the $z$-direction leads to the semi-discrete formulation

$$
\partial_{t}\left(h_{\alpha} \overline{\mathbf{R}}_{\alpha}\right)+\partial_{x}\left(h_{\alpha}\left[\overline{u \mathbf{R}}_{\alpha}+\overline{\mathbf{P}}_{\alpha}\right]\right)+\mathscr{F}_{\alpha+1 / 2}^{\mathbf{R}}-\mathscr{F}_{\alpha-1 / 2}^{\mathbf{R}}=h_{\alpha} \overline{\mathbf{S}}_{\alpha}
$$

where

$$
\mathscr{F}_{\alpha+1 / 2}^{\mathbf{R}}=\Gamma_{\alpha+1 / 2} \widetilde{\mathbf{R}}_{\alpha+1 / 2}-\widetilde{\mathbf{P}}_{\alpha+1 / 2} \partial_{x} z_{\alpha+1 / 2}+\widetilde{\mathbf{Q}}_{\alpha+1 / 2} .
$$

The average $\widetilde{\mathbf{R}}_{\alpha+1 / 2}$ is defined by the definition (3.3) and $\Gamma_{\alpha+1 / 2}$ by (3.11).

Proof: The jump condition associated to Equation (4.1) across a potential isolated discontinuity located at $z=\mathcal{Z}(t, x)$ reads

$$
\partial_{t} \mathcal{Z} \llbracket \mathbf{R} \rrbracket_{z=\mathcal{Z}}+\partial_{x} \mathcal{Z} \llbracket u \mathbf{R}+\mathbf{P} \rrbracket_{z=\mathcal{Z}}-\llbracket w \mathbf{R}+\mathbf{Q} \rrbracket_{z=\mathcal{Z}}=0
$$

or equivalently

$$
\Gamma \llbracket \mathbf{R} \rrbracket-\partial_{x} \mathcal{Z} \llbracket \mathbf{P} \rrbracket+\llbracket \mathbf{Q} \rrbracket=0
$$

where $\Gamma=w_{\mid z=\mathcal{Z}}-\partial_{t} \mathcal{Z}-u_{\mid z=\mathcal{Z}} \partial_{x} \mathcal{Z}$. Integrating Eq. (4.1) over a layer $\mathcal{L}_{\alpha}$ yields

$$
\begin{aligned}
h_{\alpha}\langle\mathbf{S}\rangle_{\alpha}= & \partial_{t}\left(h_{\alpha}\langle\mathbf{R}\rangle_{\alpha}\right)-\mathbf{R}_{\alpha+1 / 2}^{-} \partial_{t} z_{\alpha+1 / 2}+\mathbf{R}_{\alpha-1 / 2}^{+} \partial_{t} z_{\alpha-1 / 2} \\
& +\partial_{x}\left(h_{\alpha}\langle u \mathbf{R}+\mathbf{P}\rangle_{\alpha}\right)-\left(u_{\alpha+1 / 2}^{-} \mathbf{R}_{\alpha+1 / 2}^{-}+\mathbf{P}_{\alpha+1 / 2}^{-}\right) \partial_{x} z_{\alpha+1 / 2}+\left(u_{\alpha-1 / 2}^{+} \mathbf{R}_{\alpha-1 / 2}^{+}+\mathbf{P}_{\alpha-1 / 2}^{+}\right) \partial_{x} z_{\alpha-1 / 2} \\
& +w_{\alpha+1 / 2}^{-} \mathbf{R}_{\alpha+1 / 2}^{-}+\mathbf{Q}_{\alpha+1 / 2}^{-}-w_{\alpha-1 / 2}^{+} \mathbf{R}_{\alpha-1 / 2}^{+}+\mathbf{Q}_{\alpha-1 / 2}^{+} \\
= & \partial_{t}\left(h_{\alpha}\langle\mathbf{R}\rangle_{\alpha}\right)+\partial_{x}\left(h_{\alpha}\langle u \mathbf{R}+\mathbf{P}\rangle_{\alpha}\right)+\left(\mathbf{R}_{\alpha+1 / 2}^{-} \Gamma_{\alpha+1 / 2}-\mathbf{P}_{\alpha+1 / 2}^{-} \partial_{x} z_{\alpha+1 / 2}+\mathbf{Q}_{\alpha+1 / 2}^{-}\right) \\
& -\left(\mathbf{R}_{\alpha-1 / 2}^{+} \Gamma_{\alpha-1 / 2}-\mathbf{P}_{\alpha-1 / 2}^{+} \partial_{x} z_{\alpha-1 / 2}+\mathbf{Q}_{\alpha-1 / 2}^{+}\right) .
\end{aligned}
$$

Upper and lower limits between brackets can be replaced by mean values using (4.3). This leads to (4.2).

\subsubsection{Application to the Euler equations}

To derive Eqs. (3.9a), (3.9b) and (3.9c) we rewrite the Euler equations (2.5) under the system of conservation laws (4.1), which corresponds to

$$
\mathbf{R}=\left(\begin{array}{l}
1 \\
\underline{u} \\
\underline{w}
\end{array}\right), \mathbf{P}=\left(\begin{array}{l}
0 \\
\underline{q} \\
0
\end{array}\right), \mathbf{Q}=\left(\begin{array}{l}
0 \\
0 \\
\underline{q}
\end{array}\right) \text { and } \mathbf{S}=\left(\begin{array}{c}
0 \\
-\partial_{x}\left(\underline{g} \underline{\eta}+p^{a t m}\right) \\
0
\end{array}\right) .
$$

Given the $\mathbb{P}_{0}$-approximation (3.4) for $\underline{u}$, the second and third equations of the numerical scheme (4.2a) applied to (4.4) yield directly Eqs. (3.9b) and (3.9c). As for the first equation of (4.2a), it reads in the present case

$$
\partial_{t} h_{\alpha}+\partial_{x}\left(h_{\alpha} u_{\alpha}\right)=\Gamma_{\alpha-1 / 2}-\Gamma_{\alpha+1 / 2}
$$

This equation models the evolution of the mass of fluid within layer $\mathcal{L}_{\alpha}$ : on the one hand, the fluid is transported at velocity $u_{\alpha}$; on the other hand, mass is gained/lost through interfaces, i.e. from layers $\mathcal{L}_{\alpha-1}$ and $\mathcal{L}_{\alpha+1}$. Let us recall that $\Gamma_{\alpha+1 / 2}$ is a transfer term, that describes the amount of fluid that moves from $\mathcal{L}_{\alpha}$ to $\mathcal{L}_{\alpha+1}$. At the continuous level, the mass flux is defined by

$$
\underline{\Gamma}_{\alpha+1 / 2}=\underline{\tilde{\boldsymbol{u}}}_{\alpha+1 / 2} \cdot \underline{\boldsymbol{n}}_{\alpha+1 / 2}-\partial_{t} \underline{z}_{\alpha+1 / 2}=\underline{\widetilde{w}}_{\alpha+1 / 2}-\left(\partial_{t} \underline{z}_{\alpha+1 / 2}+\underline{\tilde{u}}_{\alpha+1 / 2} \partial_{x} \underline{z}_{\alpha+1 / 2}\right) .
$$

Jump condition (3.2a) enables to rewrite equally (4.6) as ${ }^{5}$

$$
\underline{\Gamma}_{\alpha+1 / 2}=\underline{w}_{\alpha+1 / 2}^{-}-\left(\partial_{t} \underline{z}_{\alpha+1 / 2}+\underline{u}_{\alpha+1 / 2}^{-} \partial_{x} \underline{z}_{\alpha+1 / 2}\right)=\underline{w}_{\alpha+1 / 2}^{+}-\left(\partial_{t} \underline{z}_{\alpha+1 / 2}+\underline{u}_{\alpha+1 / 2}^{+} \partial_{x} \underline{z}_{\alpha+1 / 2}\right) .
$$

\footnotetext{
${ }^{5}$ If coefficients specific to each unknown were used in (3.3), then (4.7) would hold provided $\gamma_{\alpha+1 / 2}^{u}=\gamma_{\alpha+1 / 2}^{w}$.
} 
Boundary conditions (2.2a) and (2.2c) imply respectively

$$
\underline{\Gamma}_{1 / 2}=0 \text { and } \underline{\Gamma}_{L+1 / 2}=0 .
$$

Then, the summation of (4.5) for $\alpha$ from 1 to $L$ leads to Eq. (3.9a). A summation for $\beta$ from 1 to $\alpha$ or from $\alpha+1$ to $L$ provides

$$
\Gamma_{\alpha+1 / 2}=\sum_{\beta=\alpha+1}^{L}\left[\partial_{t} h_{\beta}+\partial_{x}\left(h_{\beta} u_{\beta}\right)\right]=-\sum_{\beta=1}^{\alpha}\left[\partial_{t} h_{\beta}+\partial_{x}\left(h_{\beta} u_{\beta}\right)\right]
$$

This derivation only uses the $\mathbb{P}_{0}$ approximation (3.4) for $\underline{u}$.

In the approximation framework (3.7), $w$ is a 1st-order polynomial with respect to $z$ within each layer. Let us focus on interface values for $w$. (3.9e) is the discrete counterpart to the left hand side of (3.12). As for the right hand side, it is deduced from

$$
\begin{aligned}
w_{\alpha+1 / 2}^{-}-w_{\alpha-1 / 2}^{+}=\partial_{t} z_{\alpha+1 / 2}+u_{\alpha} \partial_{x} z_{\alpha+1 / 2}+\Gamma_{\alpha+1 / 2}-\partial_{t} z_{\alpha-1 / 2}-u_{\alpha} \partial_{x} z_{\alpha-1 / 2}-\Gamma_{\alpha-1 / 2} \\
\stackrel{(4.9)}{=} \partial_{t} h_{\alpha}+u_{\alpha} \partial_{x} h_{\alpha}-\left[\partial_{t} h_{\alpha}+\partial_{x}\left(h_{\alpha} u_{\alpha}\right)\right]=-h_{\alpha} \partial_{x} u_{\alpha} .
\end{aligned}
$$

We come to the conclusion combining this result with (3.9e). We thus have

$$
w_{\alpha+1 / 2}^{-}=w_{\alpha}-\frac{h_{\alpha} \partial_{x} u_{\alpha}}{2} \quad \text { and } \quad w_{\alpha-1 / 2}^{+}=w_{\alpha}+\frac{h_{\alpha} \partial_{x} u_{\alpha}}{2} .
$$

To fully determine this velocity component, an additional equation is required, for instance by projecting the momentum equation $(2.5 \mathrm{c})$ onto the basis $(1, z)$. More precisely, multiplying $(2.5 \mathrm{c})$ by $z$, we obtain

$$
\partial_{t}(z \underline{w})+\partial_{x}(z \underline{u w})+\partial_{z}\left(z\left(\underline{w}^{2}+\underline{q}\right)\right)=\underline{w}^{2}+\underline{q} .
$$

We then apply the semi-discrete approximation (4.2). Lower and upper limits $w_{\alpha+1 / 2}^{-}$and $w_{\alpha-1 / 2}^{+}$are prescribed by (4.10). As $w$ is layerwise affine, the averaging process requires to compute mean values of quadratic polynomials, namely $z w$ and $w^{2}$. In view of (3.5), we have

$$
\left\{\begin{array}{l}
\langle z \underline{w}\rangle_{\alpha}=z_{\alpha} w_{\alpha}-\frac{h_{\alpha}^{2} \partial_{x} u_{\alpha}}{12}+\mathcal{O}(\varepsilon), \\
(z \underline{w})_{\alpha+1 / 2}=z_{\alpha+1 / 2} \widetilde{w}_{\alpha+1 / 2}+\mathcal{O}(\varepsilon), \\
\left\langle\underline{w}^{2}\right\rangle_{\alpha}=w_{\alpha}^{2}+\frac{h_{\alpha}^{2}\left(\partial_{x} u_{\alpha}\right)^{2}}{12}+\mathcal{O}(\varepsilon) .
\end{array}\right.
$$

Using the definition (3.9g) for $\sigma_{\alpha}$, the application of (4.2a) to (4.11) combined with (4.12) reads

$$
\begin{aligned}
& \partial_{t}\left(h_{\alpha} z_{\alpha} w_{\alpha}\right)+\partial_{x}\left(h_{\alpha} z_{\alpha} u_{\alpha} w_{\alpha}\right)+\partial_{t}\left(\frac{h_{\alpha}^{2} \sigma_{\alpha}}{2 \sqrt{3}}\right)+\partial_{x}\left(\frac{h_{\alpha}^{2} \sigma_{\alpha} u_{\alpha}}{2 \sqrt{3}}\right) \\
& \quad+z_{\alpha+1 / 2}\left(\widetilde{w}_{\alpha+1 / 2} \Gamma_{\alpha+1 / 2}+q_{\alpha+1 / 2}\right)-z_{\alpha-1 / 2}\left(\widetilde{w}_{\alpha-1 / 2} \Gamma_{\alpha-1 / 2}+q_{\alpha-1 / 2}\right)=h_{\alpha}\left(w_{\alpha}^{2}+\sigma_{\alpha}^{2}+q_{\alpha}\right) .
\end{aligned}
$$

According to (4.7) and (4.10), we notice that

$$
\partial_{t} z_{\alpha}+u_{\alpha} \partial_{x} z_{\alpha}=w_{\alpha}-\frac{\Gamma_{\alpha+1 / 2}+\Gamma_{\alpha-1 / 2}}{2} .
$$

Hence, thanks to (4.5) and (3.9c), we conclude that smooth solutions to Eq. (4.13) also satisfy Eq. (3.9d). 


\subsection{Energy}

Let us show that the dispersive models presented in Section 3.2 satisfy an energy estimate. We first recall the energy equality at the continuous level. The kinetic energy is denoted by

$$
\underline{\mathcal{K}}=\frac{\underline{u}^{2}+\underline{w}^{2}}{2} .
$$

Then for $(\underline{u}, \underline{w}, \underline{p})$ smooth solutions to the Euler equations (2.1), we have

$$
\begin{aligned}
& \partial_{t}\left(\int_{z_{b}}^{\underline{\eta}}\left(\underline{\mathcal{K}}+g \frac{\underline{\eta}+z_{b}}{2}+p^{a t m}\right) \mathrm{d} z\right)+\partial_{x}\left(\int_{z_{b}}^{\underline{\eta}} \underline{u}\left(\underline{\mathcal{K}}+\underline{q}+g \underline{\eta}+p^{a t m}\right) \mathrm{d} z\right) \\
&=\underline{H} \partial_{t} p^{a t m}+\left(g \underline{H}+\underline{q}_{\mid z=z_{b}}\right) \partial_{t} z_{b} .
\end{aligned}
$$

Let us now turn to the semi-discrete level and set

$$
\overline{\mathcal{K}}_{\alpha}=\frac{u_{\alpha}^{2}+w_{\alpha}^{2}+\sigma_{\alpha}^{2}}{2} .
$$

\section{Proposition 1}

Let us assume that

$$
\left(\gamma_{\alpha+1 / 2}-\frac{1}{2}\right) \Gamma_{\alpha+1 / 2} \geq 0
$$

If $\left(H, u_{\alpha}, w_{\alpha}, q_{\alpha}\right)$ are smooth solutions to (3.9), we have

$$
\begin{aligned}
\partial_{t}\left(\sum_{\alpha=1}^{L} h_{\alpha}\left(\overline{\mathcal{K}}_{\alpha}+g z_{\alpha}+p^{a t m}\right)\right)+\partial_{x}\left(\sum _ { \alpha = 1 } ^ { L } h _ { \alpha } u _ { \alpha } \left(\overline{\mathcal{K}}_{\alpha}+q_{\alpha}+g \eta\right.\right. & \left.\left.+p^{a t m}\right)\right) \\
& \leq H \partial_{t} p^{a t m}+\left(g H+q_{1 / 2}\right) \partial_{t} z_{b}
\end{aligned}
$$

Moreover, if we take $\gamma_{\alpha+1 / 2}=\frac{1}{2}$, then (4.17) is an equality.

Remark 2 Due to the identity

$$
\sum_{\alpha=1}^{L} h_{\alpha} z_{\alpha}=\sum_{\alpha=1}^{L} \frac{z_{\alpha+1 / 2}^{2}-z_{\alpha-1 / 2}^{2}}{2}=\frac{z_{L+1 / 2}^{2}-z_{1 / 2}^{2}}{2}=\int_{z_{b}}^{\eta} \frac{\eta+z_{b}}{2},
$$

we notice that (4.17) is the exact discrete counterpart of the continuous estimate (4.14) in the case $\gamma_{\alpha+1 / 2}=$ $\frac{1}{2}$. Otherwise, the system turns out to dissipate energy.

Remark 3 Constraint (4.16) is equivalent to taking ${ }^{6}$

$$
\gamma_{\alpha+1 / 2}=\frac{1}{2}\left(1+\Upsilon \operatorname{sign}\left(\Gamma_{\alpha+1 / 2}\right)\right)
$$

for any $\Upsilon \geq 0$. Consequently, for $\widetilde{\mathbf{R}}_{\alpha+1 / 2}$ defined by (3.3), we have

$$
\widetilde{\mathbf{R}}_{\alpha+1 / 2} \Gamma_{\alpha+1 / 2}=\frac{\mathbf{R}_{\alpha+1 / 2}^{+}+\mathbf{R}_{\alpha+1 / 2}^{-}}{2} \Gamma_{\alpha+1 / 2}-\frac{\Upsilon}{2}\left|\Gamma_{\alpha+1 / 2}\right|\left(\mathbf{R}_{\alpha+1 / 2}^{+}-\mathbf{R}_{\alpha+1 / 2}^{-}\right) .
$$

We recover the standard Rusanov flux type for finite volume schemes. (4.16) is satisfied in particular for $\gamma_{\alpha+1 / 2}=\frac{1}{2}\left(\Upsilon=0\right.$ in (4.19)) [24,25] and for $\gamma_{\alpha+1 / 2}=\mathbb{1}_{\left\{\Gamma_{\alpha+1 / 2} \geq 0\right\}}(\Upsilon=1$ in (4.19)) [5].

\footnotetext{
${ }^{6}$ The function sign is such that $x \operatorname{sign}(x)=|x|$.
} 
Remark 4 The statement of Prop. 1 stands for the $\mathrm{LDNH}_{2}$-model. Energy inequalities for Models $\mathrm{LDNH}_{1}$ and $\mathrm{LDNH}_{0}$ are put in Appendices $B$ and $C$ where the kinetic energy $\overline{\mathcal{K}}_{\alpha}$ reduces to

$$
\bar{K}_{\alpha}=\frac{u_{\alpha}^{2}+w_{\alpha}^{2}}{2} .
$$

due to the approximation made in the latter models.

Proof of Prop. 1 : Multiplying respectively (3.9b) by $u_{\alpha},(3.9 \mathrm{c})$ by $w_{\alpha}$ and (3.9d) by $\sigma_{\alpha}$ leads to

$$
\begin{aligned}
& \partial_{t}\left(h_{\alpha} \frac{u_{\alpha}^{2}}{2}\right)+\partial_{x}\left(h_{\alpha} u_{\alpha}\left(\frac{u_{\alpha}^{2}}{2}+q_{\alpha}+g \eta+p^{a t m}\right)\right)+u_{\alpha}\left(\widetilde{u}_{\alpha+1 / 2} \Gamma_{\alpha+1 / 2}-\widetilde{u}_{\alpha-1 / 2} \Gamma_{\alpha-1 / 2}\right) \\
&-u_{\alpha}\left(\partial_{x} z_{\alpha+1 / 2} q_{\alpha+1 / 2}-\partial_{x} z_{\alpha-1 / 2} q_{\alpha-1 / 2}\right)=h_{\alpha} q_{\alpha} \partial_{x} u_{\alpha}+\frac{u_{\alpha}^{2}}{2}\left(\Gamma_{\alpha+1 / 2}-\Gamma_{\alpha-1 / 2}\right)+\left(g \eta+p^{a t m}\right) \partial_{x}\left(h_{\alpha} u_{\alpha}\right), \\
& \partial_{t}\left(h_{\alpha} \frac{w_{\alpha}^{2}}{2}\right)+\partial_{x}\left(h_{\alpha} u_{\alpha} \frac{w_{\alpha}^{2}}{2}\right)+w_{\alpha}\left(\widetilde{w}_{\alpha+1 / 2} \Gamma_{\alpha+1 / 2}+q_{\alpha+1 / 2}-\widetilde{w}_{\alpha-1 / 2} \Gamma_{\alpha-1 / 2}-q_{\alpha-1 / 2}\right)=\frac{w_{\alpha}^{2}}{2}\left(\Gamma_{\alpha+1 / 2}-\Gamma_{\alpha-1 / 2}\right), \\
& \partial_{t}\left(h_{\alpha} \frac{\sigma_{\alpha}^{2}}{2}\right)+\partial_{x}\left(h_{\alpha} u_{\alpha} \frac{\sigma_{\alpha}^{2}}{2}\right)= \frac{\sigma_{\alpha}^{2}}{2}\left(\Gamma_{\alpha+1 / 2}-\Gamma_{\alpha-1 / 2}\right)+2 \sqrt{3} \sigma_{\alpha}\left[q_{\alpha}-\frac{q_{\alpha+1 / 2}+q_{\alpha-1 / 2}}{2}\right. \\
&\left.-\Gamma_{\alpha+1 / 2}\left(\frac{h_{\alpha} \partial_{x} u_{\alpha}}{12}+\frac{\widetilde{w}_{\alpha+1 / 2}-w_{\alpha}}{2}\right)+\Gamma_{\alpha-1 / 2}\left(\frac{h_{\alpha} \partial_{x} u_{\alpha}}{12}+\frac{w_{\alpha}-\widetilde{w}_{\alpha-1 / 2}}{2}\right)\right],
\end{aligned}
$$

Let us sum the three equalities

$$
\begin{aligned}
\partial_{t}\left(h_{\alpha} \overline{\mathcal{K}}_{\alpha}\right)+ & \partial_{x}\left(h_{\alpha} u_{\alpha}\left[\overline{\mathcal{K}}_{\alpha}+q_{\alpha}+g \eta+p^{a t m}\right]\right)=\left(g \eta+p^{a t m}\right) \partial_{x}\left(h_{\alpha} u_{\alpha}\right) \\
& +q_{\alpha+1 / 2}\left(u_{\alpha} \partial_{x} z_{\alpha+1 / 2}-w_{\alpha}-\sqrt{3} \sigma_{\alpha}\right)-q_{\alpha-1 / 2}\left(u_{\alpha} \partial_{x} z_{\alpha-1 / 2}-w_{\alpha}+\sqrt{3} \sigma_{\alpha}\right) \\
& -\Gamma_{\alpha+1 / 2}\left(u_{\alpha} \widetilde{u}_{\alpha+1 / 2}+w_{\alpha} \widetilde{w}_{\alpha+1 / 2}+\left(\frac{\widetilde{w}_{\alpha+1 / 2}-w_{\alpha}}{2}-\frac{\sigma_{\alpha}}{2 \sqrt{3}}\right) 2 \sqrt{3} \sigma_{\alpha}-\overline{\mathcal{K}}_{\alpha}\right) \\
& +\Gamma_{\alpha-1 / 2}\left(u_{\alpha} \widetilde{u}_{\alpha-1 / 2}+w_{\alpha} \widetilde{w}_{\alpha-1 / 2}+\left(\frac{w_{\alpha}-\widetilde{w}_{\alpha-1 / 2}}{2}-\frac{\sigma_{\alpha}}{2 \sqrt{3}}\right) 2 \sqrt{3} \sigma_{\alpha}-\overline{\mathcal{K}}_{\alpha}\right) .
\end{aligned}
$$

On the one hand, we focus on pressure terms. We notice that

$$
u_{\alpha} \partial_{x} z_{\alpha+1 / 2}-w_{\alpha}-\sqrt{3} \sigma_{\alpha} \stackrel{(3.9 \mathrm{e})}{=} u_{\alpha} \partial_{x} z_{\alpha+1 / 2}-w_{\alpha+1 / 2}^{-}=-\Gamma_{\alpha+1 / 2}-\partial_{t} z_{\alpha+1 / 2} .
$$

Hence the sum over all layers is equal to

$$
-\sum_{\alpha=1}^{L}\left[q_{\alpha+1 / 2}\left(\Gamma_{\alpha+1 / 2}+\partial_{t} z_{\alpha+1 / 2}\right)-q_{\alpha-1 / 2}\left(\Gamma_{\alpha-1 / 2}+\partial_{t} z_{\alpha-1 / 2}\right)\right]=q_{1 / 2} \partial_{t} z_{b}
$$

according to boundary conditions (3.10b) and (4.8). On the other hand, we consider terms involving $\Gamma_{\alpha+1 / 2}$ in (4.21). We have

$$
-\left(\frac{w_{\alpha}}{2}+\frac{\sigma_{\alpha}}{2 \sqrt{3}}\right) 2 \sqrt{3} \sigma_{\alpha}-\frac{w_{\alpha}^{2}+\sigma_{\alpha}^{2}}{2}=-\frac{\left(w_{\alpha+1 / 2}^{-}\right)^{2}}{2} .
$$

If we sum (4.21) for $\alpha$ from 1 to $L$, we obtain thanks to (3.9a)

$$
\begin{array}{r}
\partial_{t}\left(\sum_{\alpha=1}^{L} h_{\alpha} \overline{\mathcal{K}}_{\alpha}\right)+\partial_{x}\left(\sum_{\alpha=1}^{L} h_{\alpha} u_{\alpha}\left(\overline{\mathcal{K}}_{\alpha}+q_{\alpha}+g \eta+p^{a t m}\right)\right)=-\left(g \eta+p^{a t m}\right) \partial_{t} H+q_{1 / 2} \partial_{t} z_{b} \\
-\sum_{\alpha=1}^{L} \Gamma_{\alpha+1 / 2}\left[u_{\alpha}\left(\widetilde{u}_{\alpha+1 / 2}-\frac{u_{\alpha}}{2}\right)+w_{\alpha+1 / 2}^{-}\left(\widetilde{w}_{\alpha+1 / 2}-\frac{w_{\alpha+1 / 2}^{-}}{2}\right)\right] \\
-\Gamma_{\alpha-1 / 2}\left[u_{\alpha}\left(\widetilde{u}_{\alpha-1 / 2}-\frac{u_{\alpha}}{2}\right)+w_{\alpha-1 / 2}^{+}\left(\widetilde{w}_{\alpha-1 / 2}-\frac{w_{\alpha-1 / 2}^{+}}{2}\right)\right] .
\end{array}
$$

On the one hand, we notice that

$$
\eta \partial_{t} H=\eta \partial_{t} \eta-\eta \partial_{t} z_{b}=\partial_{t}\left(\frac{\eta^{2}-z_{b}^{2}}{2}\right)-H \partial_{t} z_{b} \stackrel{(4.18)}{=} \partial_{t}\left(\sum_{\alpha=1}^{L} h_{\alpha} z_{\alpha}\right)-H \partial_{t} z_{b} .
$$


On the other hand we get by a change of indices

$$
\begin{aligned}
\sum_{\alpha=1}^{L}\left[\Gamma _ { \alpha + 1 / 2 } u _ { \alpha } \left(\widetilde{u}_{\alpha+1 / 2}\right.\right. & \left.\left.-\frac{u_{\alpha}}{2}\right)-\Gamma_{\alpha-1 / 2} u_{\alpha}\left(\widetilde{u}_{\alpha-1 / 2}-\frac{u_{\alpha}}{2}\right)\right] \\
& =-\sum_{\alpha=1}^{L-1} \Gamma_{\alpha+1 / 2}\left(\widetilde{u}_{\alpha+1 / 2}-\frac{u_{\alpha}+u_{\alpha+1}}{2}\right) \llbracket u \rrbracket_{\alpha+1 / 2} \stackrel{(3.3)}{=} \sum_{\alpha=1}^{L-1} \Gamma_{\alpha+1 / 2}\left(\gamma_{\alpha+1 / 2}-\frac{1}{2}\right) \llbracket u \rrbracket_{\alpha+1 / 2}^{2} .
\end{aligned}
$$

Likewise

$$
\sum_{\alpha=1}^{L}\left[\Gamma_{\alpha+1 / 2} w_{\alpha}\left(\widetilde{w}_{\alpha+1 / 2}-\frac{w_{\alpha+1 / 2}^{-}}{2}\right)-\Gamma_{\alpha-1 / 2} w_{\alpha}\left(\widetilde{w}_{\alpha-1 / 2}-\frac{w_{\alpha-1 / 2}^{+}}{2}\right)\right]=\sum_{\alpha=1}^{L-1} \Gamma_{\alpha+1 / 2}\left(\gamma_{\alpha+1 / 2}-\frac{1}{2}\right) \llbracket w \rrbracket_{\alpha+1 / 2}^{2} .
$$

In conclusion, given the latter equalities, (4.22) reduces to

$$
\begin{aligned}
\partial_{t}\left(\sum_{\alpha=1}^{L} h_{\alpha}\left(\overline{\mathcal{K}}_{\alpha}+g z_{\alpha}+p^{a t m}\right)\right) & \partial_{x}\left(\sum_{\alpha=1}^{L} h_{\alpha} u_{\alpha}\left(\overline{\mathcal{K}}_{\alpha}+q_{\alpha}+g \eta+p^{a t m}\right)\right) \\
& =H \partial_{t} p^{a t m}+\left(g H+q_{1 / 2}\right) \partial_{t} z_{b}-\sum_{\alpha=1}^{L-1} \Gamma_{\alpha+1 / 2}\left(\gamma_{\alpha+1 / 2}-\frac{1}{2}\right)\left[1+\left(\partial_{x} z_{\alpha+1 / 2}\right)^{2}\right] \llbracket u \rrbracket_{\alpha+1 / 2}^{2}
\end{aligned}
$$

where we used jump conditions (3.10a). Then (4.23) implies Estimate (4.17) provided that (4.16) holds which ensures the negativity of the sum in the right hand side. In the particular case where $\gamma_{\alpha+1 / 2}=\frac{1}{2}$, this sum vanishes and (4.17) holds as an equality.

\subsection{Hydrodynamic balances}

Classically, the integration over the whole domain $\Omega(t)$ of the three Euler equations (2.1) ensures the following equalities, provided solutions are smooth and integrable:

- The conservation of global volume:

$$
\partial_{t}\left(\int_{\mathbb{R}} \underline{H}(t, x) \mathrm{d} x\right)=0
$$

- The balance of horizontal momentum:

$$
\begin{aligned}
\partial_{t}\left(\int_{\mathbb{R}}\left(\int_{z_{b}(t, x)}^{\underline{\eta}(t, x)} \underline{u}(t, x, z) \mathrm{d} z\right) \mathrm{d} x\right) \\
\quad=-\int_{\mathbb{R}}\left(\underline{H}(t, x) \partial_{x} p^{a t m}(t, x)+\left(g \underline{H}(t, x)+\underline{q}\left(t, x, z_{b}(t, x)\right)\right) \partial_{x} z_{b}(t, x)\right) \mathrm{d} x
\end{aligned}
$$

- The balance of vertical momentum:

$$
\partial_{t}\left(\int_{\mathbb{R}}\left(\int_{z_{b}(t, x)}^{\underline{\eta}(t, x)} \underline{w}(t, x, z) \mathrm{d} z\right) \mathrm{d} x\right)=\int_{\mathbb{R}} \underline{q}\left(t, x, z_{b}(t, x)\right) \mathrm{d} x .
$$

At the discrete level, smooth solutions to the $\mathrm{LDNH}_{2}$ model satisfy similar balance equations. 


\section{Proposition 2}

Let $\left(H, u_{\alpha}, w_{\alpha}, q_{\alpha}\right)$ be smooth solutions to (3.9) and (3.10). Then the following equalities hold:

- The conservation of global volume:

$$
\partial_{t}\left(\int_{\mathbb{R}} H(t, x) \mathrm{d} x\right)=0
$$

- The balance of horizontal momentum:

$$
\partial_{t}\left(\int_{\mathbb{R}} H(t, x) \bar{u}(t, x) \mathrm{d} x\right)=-\int_{\mathbb{R}}\left(H(t, x) \partial_{x} p^{a t m}(t, x)+\left(g H(t, x)+q_{1 / 2}(t, x)\right) \partial_{x} z_{b}(x)\right) \mathrm{d} x ;
$$

- The balance of vertical momentum:

$$
\partial_{t}\left(\int_{\mathbb{R}} H(t, x) \bar{w}(t, x) \mathrm{d} x\right)=-\int_{\mathbb{R}} q_{1 / 2}(t, x) \mathrm{d} x .
$$

Proof: The conservation law (4.25a) results from the direct integration of Eq. (3.9a). We also notice that according to boundary conditions $(3.10 \mathrm{~b})$ and $(4.8)$ we have

$$
\sum_{\alpha=1}^{L}\left(\Gamma_{\alpha+1 / 2}\left(\begin{array}{c}
\widetilde{u}_{\alpha+1 / 2} \\
\widetilde{w}_{\alpha+1 / 2}
\end{array}\right)+q_{\alpha+1 / 2} \boldsymbol{n}_{\alpha+1 / 2}-\Gamma_{\alpha-1 / 2}\left(\begin{array}{c}
\widetilde{u}_{\alpha-1 / 2} \\
\widetilde{w}_{\alpha-1 / 2}
\end{array}\right)-q_{\alpha-1 / 2} \boldsymbol{n}_{\alpha-1 / 2}\right)=-q_{1 / 2} \boldsymbol{n}_{1 / 2} .
$$

Hence summing Eq. (3.9b) over $\alpha$ leads to

$$
\begin{aligned}
\partial_{t}\left(\int_{\mathbb{R}} H \bar{u}\right) & =\int_{\mathbb{R}} \partial_{t}\left(\sum_{\alpha=1}^{L} h_{\alpha} u_{\alpha}\right)=\int_{\mathbb{R}}-q_{1 / 2} \partial_{x} z_{b}-\sum_{\alpha=1}^{L}\left[\partial_{x}\left(h_{\alpha} u_{\alpha}^{2}+h_{\alpha} q_{\alpha}\right)+h_{\alpha} \partial_{x}\left(g \eta+p^{a t m}\right)\right] \\
& =-\int_{\mathbb{R}}\left(q_{1 / 2} \partial_{x} z_{b}+H \partial_{x} p^{a t m}+g \partial_{x}\left(\frac{H^{2}}{2}\right)+g H \partial_{x} z_{b}\right)
\end{aligned}
$$

which proves $(4.25 \mathrm{~b})$. Similarly the integration of $(2.5 \mathrm{c})$ gives

$$
\partial_{t}\left(\int_{\mathbb{R}} H \bar{w}\right)=\int_{\mathbb{R}} \partial_{t}\left(\sum_{\alpha=1}^{L} h_{\alpha} w_{\alpha}\right)=\int_{\mathbb{R}} q_{1 / 2}-\sum_{\alpha=1}^{L} \partial_{x}\left(h_{\alpha} u_{\alpha} w_{\alpha}\right)=\int_{\mathbb{R}} q_{1 / 2}
$$

and $(4.25 \mathrm{c})$ is proven.

The same balances - which are the discrete counterparts of (4.24) - hold for Models $\mathrm{LDNH}_{1}$ and $\mathrm{LDNH}_{0}$.

\subsection{Dispersion relations}

We first mention that the so-called lake-at-rest state is a steady solution for all the models described in the present paper.

Remark 5 For a given constant $\bar{\phi}$ such that $\bar{\phi} \geq g z_{b}+p^{a t m}$, then

$$
\left(H_{0}=-z_{b}+\frac{\bar{\phi}-p^{a t m}}{g}, u_{\alpha}=0, w_{\alpha}=0, q_{\alpha}=0\right)
$$

is a steady state for Models $\mathrm{LDNH}_{2}, \mathrm{LDNH}_{1}$ and $\mathrm{LDNH}_{0}$. 


\subsubsection{Main statement}

For the sake of simplicity, this section is restricted to the case where $z_{b}$ and $p^{a t m}$ are constant in time and space.

Let us linearise the $\mathrm{LDNH}_{2}$ model around the steady state described in Lemma $5\left(H_{0}, u_{0}=0, w_{0}=0, q_{0}=0\right)$ for any $H_{0}>0$. It comes down to considering asymptotic expansions

$$
\star=\star^{(0)}+\epsilon \star^{(1)}+\mathcal{O}\left(\epsilon^{2}\right), \quad \text { for } \epsilon \ll 1 \text { and } \star^{(0)}=\star_{0},
$$

that are substituted into Eqs. (3.9)-(3.10). The resulting model at order 1 reads

$$
\left\{\begin{array}{l}
\partial_{t} H^{(1)}+H_{0} \sum_{\alpha=1}^{L} \ell_{\alpha} \partial_{x} u_{\alpha}^{(1)}=0 \\
\partial_{t} u_{\alpha}^{(1)}+\partial_{x} q_{\alpha}^{(1)}+g \partial_{x} H^{(1)}=0 \\
\ell_{\alpha} H_{0} \partial_{t} w_{\alpha}^{(1)}+q_{\alpha+1 / 2}^{(1)}-q_{\alpha-1 / 2}^{(1)}=0 \\
\ell_{\alpha}^{2} H_{0}^{2} \partial_{t x}^{2} u_{\alpha}^{(1)}+12\left(q_{\alpha}^{(1)}-\frac{q_{\alpha+1 / 2}^{(1)}+q_{\alpha-1 / 2}^{(1)}}{2}\right)=0 \\
w_{\alpha}^{(1)}=\frac{\ell_{\alpha}}{2} H_{0} \partial_{x} u_{\alpha}^{(1)}-H_{0} \sum_{\beta=1}^{\alpha} \ell_{\beta} \partial_{x} u_{\beta}^{(1)} .
\end{array}\right.
$$

Such a procedure can apply similarly to Models $\mathrm{LDNH}_{1}$ and $\mathrm{LDNH}_{0}$. The dispersion relations associated to those linearised systems are given in the following statement.

\section{Lemma 1}

There exists a plane wave solution $\left(\hat{H}, \hat{u}_{\alpha}, \hat{w}_{\alpha}, \hat{q}_{\alpha}\right) e^{i(k x-\omega t)}$ to the linearised version of $\left(\mathrm{LDNH}_{\nu}\right)_{\nu \in\{0,1,2\}}$ provided the following dispersion relation holds

$$
\omega^{2}=k^{2} c_{s w}^{2}\left\langle\mathcal{A}_{k H_{0}}^{-1} \boldsymbol{e}, \ell\right\rangle
$$

where $c_{s w}=\sqrt{g H_{0}}, \ell=\left(\ell_{1}, \ldots, \ell_{L}\right) \in \mathbb{R}^{L}, \boldsymbol{e}=(1, \ldots, 1) \in \mathbb{R}^{L}$ and

$$
\mathcal{A}_{x}=\mathcal{I}_{L}+x^{2} \mathcal{B}, \quad \text { with } \quad \mathcal{B}_{\alpha \beta}=-\frac{\ell_{\alpha}^{2}}{2 \lambda} \delta_{\alpha \beta}+\ell_{\beta}\left(\frac{\ell_{\max \{\alpha, \beta\}}}{2}+\sum_{\gamma=\max \{\alpha, \beta\}+1}^{L} \ell_{\gamma}\right),
$$

with $\lambda=2+\left\lfloor\frac{\nu}{2}\right\rfloor= \begin{cases}3, & \text { if } \nu=2, \\ 2, & \text { if } \nu \in\{0,1\} .\end{cases}$

The proof is detailed in Appendix A.1.

Remark 6 For characteristic numbers $k H_{0}$ small enough, matrix $\mathcal{A}_{k H_{0}}$ is invertible as a perturbation of the identity matrix. In fact for any $x, \operatorname{det} \mathcal{A}_{x} \neq 0$ which can be deduced from the same procedure as in the proof given in Appendix A.2.

Relation (4.27) is implicit and it is not of particular interest as such. When all layers have the same thickness (the choice of $\left(\ell_{\alpha}\right)$ is left to the scientist), this can be improved by means of an explicit formula. 


\section{Theorem 1}

For a given number of layers $L \geq 4$, the dispersion relation (4.27) can be made explicit in the homogeneous case $\left(\ell_{\alpha}=\frac{1}{L}\right)$

$$
c_{L}^{2}\left(k H_{0}\right)=\frac{\omega^{2}}{k^{2} g H_{0}}=\frac{\mathcal{P}_{L}\left(k H_{0}\right)}{\mathcal{Q}_{L}\left(k H_{0}\right)}
$$

where

$$
\begin{aligned}
\mathcal{P}_{L}(x)= & \frac{1}{L}\left[\left(1-\frac{x^{2}}{2 \lambda L^{2}}\right)^{L-1}+\xi_{L-4}\left(1-\frac{x^{2}}{2 \lambda L^{2}}\right)^{2}-\xi_{L-3}\left(1+\frac{2 \lambda-1}{2 \lambda} \frac{x^{2}}{L^{2}}\right)\right] \\
\mathcal{Q}_{L}(x)= & \left(1-\frac{x^{2}}{2 \lambda L^{2}}\right)^{L-1}\left(1+\frac{\lambda-1}{2 \lambda} \frac{x^{2}}{L^{2}}\right)+\left(1-\frac{x^{2}}{2 \lambda L^{2}}\right)^{2} \frac{x^{2} \xi_{L-4}}{2 L^{2}}-\left(3+\frac{2 \lambda-3}{2 \lambda} \frac{x^{2}}{L^{2}}\right) \frac{x^{2} \xi_{L-3}}{2 L^{2}} \\
\xi_{k}= & \frac{L^{2}}{x^{2}}\left(1-\frac{x^{2}}{2 \lambda L^{2}}\right)^{k+2}+\Xi_{e} \sum_{0 \leq 2 m \leq k}\left(\begin{array}{c}
k \\
2 m
\end{array}\right)\left(1+\frac{\lambda-1}{2 \lambda} \frac{x^{2}}{L^{2}}\right)^{k-2 m} \frac{x^{2 m-1}}{L^{2 m-1}}\left(1+\frac{\lambda-2}{4 \lambda} \frac{x^{2}}{L^{2}}\right)^{m} \\
& +\Xi_{o} \sum_{0 \leq 2 m+1 \leq k}\left(\begin{array}{c}
k \\
2 m+1
\end{array}\right)\left(1+\frac{\lambda-1}{2 \lambda} \frac{x^{2}}{L^{2}}\right)^{k-2 m-1} \frac{x^{2 m+1}}{L^{2 m+1}}\left(1+\frac{\lambda-2}{4 \lambda} \frac{x^{2}}{L^{2}}\right)^{m}
\end{aligned}
$$

where $\Xi_{e}=-1+\frac{1-3 \lambda}{\lambda} \frac{x^{2}}{L^{2}}+\frac{-1+6 \lambda-4 \lambda^{2}}{4 \lambda^{2}} \frac{x^{4}}{L^{4}}$ and $\Xi_{o}=-\frac{5}{2}+\frac{5(1-\lambda)}{2 \lambda} \frac{x^{2}}{L^{2}}+\frac{-\frac{5}{2}+5 \lambda-2 \lambda^{2}}{4 \lambda^{2}} \frac{x^{4}}{L^{4}}$.

Moreover, when the number $L$ of layers increases, the celerity $c_{L}$ converges to the celerity associated to the Euler equations obtained from the Airy wave theory:

$$
c_{\text {Airy }}^{2}\left(k H_{0}\right)=\frac{\tanh \left(k H_{0}\right)}{k H_{0}} .
$$

The proof is given in Appendix A.2.

Remark 7 As $\xi_{k}=-\frac{(k+3)(k+2)}{2}+\mathcal{O}\left(x^{2}\right)$, we check that $\mathcal{P}_{L}$ and $\mathcal{Q}_{L}$ are actually polynomials (w.r.t. $\left.x^{2}\right)$ of the form $1+\mathcal{O}\left(x^{2}\right)$.

For $L \leq 3$, dispersion relations are also expressed by (4.29) with:

\begin{tabular}{ccc}
\hline$L$ & $\mathcal{P}_{L}$ & $\mathcal{Q}_{L}$ \\
\hline 1 & 1 & $1+\frac{x^{2}}{3}$ \\
\hline 2 & $1+\frac{x^{2}}{12}$ & $1+\frac{5 x^{2}}{12}+\frac{7 x^{4}}{576}$ \\
\hline 3 & $1+\frac{x^{2}}{9}+\frac{5 x^{4}}{2916}$ & $1+\frac{4 x^{2}}{9}+\frac{19 x^{4}}{972}+\frac{13 x^{6}}{78732}$ \\
\hline
\end{tabular}

(a) $\mathrm{LDNH}_{2}(\lambda=3)$

\begin{tabular}{ccc}
\hline$L$ & $\mathcal{P}_{L}$ & $\mathcal{Q}_{L}$ \\
\hline 1 & 1 & $1+\frac{x^{2}}{4}$ \\
\hline 2 & $1+\frac{x^{2}}{16}$ & $1+\frac{3 x^{2}}{8}+\frac{x^{4}}{256}$ \\
\hline 3 & $1+\frac{5 x^{2}}{54}+\frac{x^{4}}{1296}$ & $1+\frac{5 x^{2}}{12}+\frac{5 x^{4}}{432}+\frac{x^{6}}{46656}$ \\
\hline
\end{tabular}

(b) $\mathrm{LDNH}_{1,0}(\lambda=2)$

Table 1: Dispersion relations for small numbers of layers

For $L=1$ in Table 1(a), we recover the classical dispersion relation for Green-Nagdhi [29] and Peregrine [44] models. We mention that data in Table $1(\mathrm{~b})$ were given in $[6,7]$.

Remark 8 To our knowledge, there is no such explicit formula as (4.29) in the literature. In [7], a qualitative result is given with determinants of extracted matrices and an asymptotic behaviour for deep water flows. In the present case, not only does the formula enable to compute an explicit quantity but it also provides a hint of convergence to the Euler equations. 


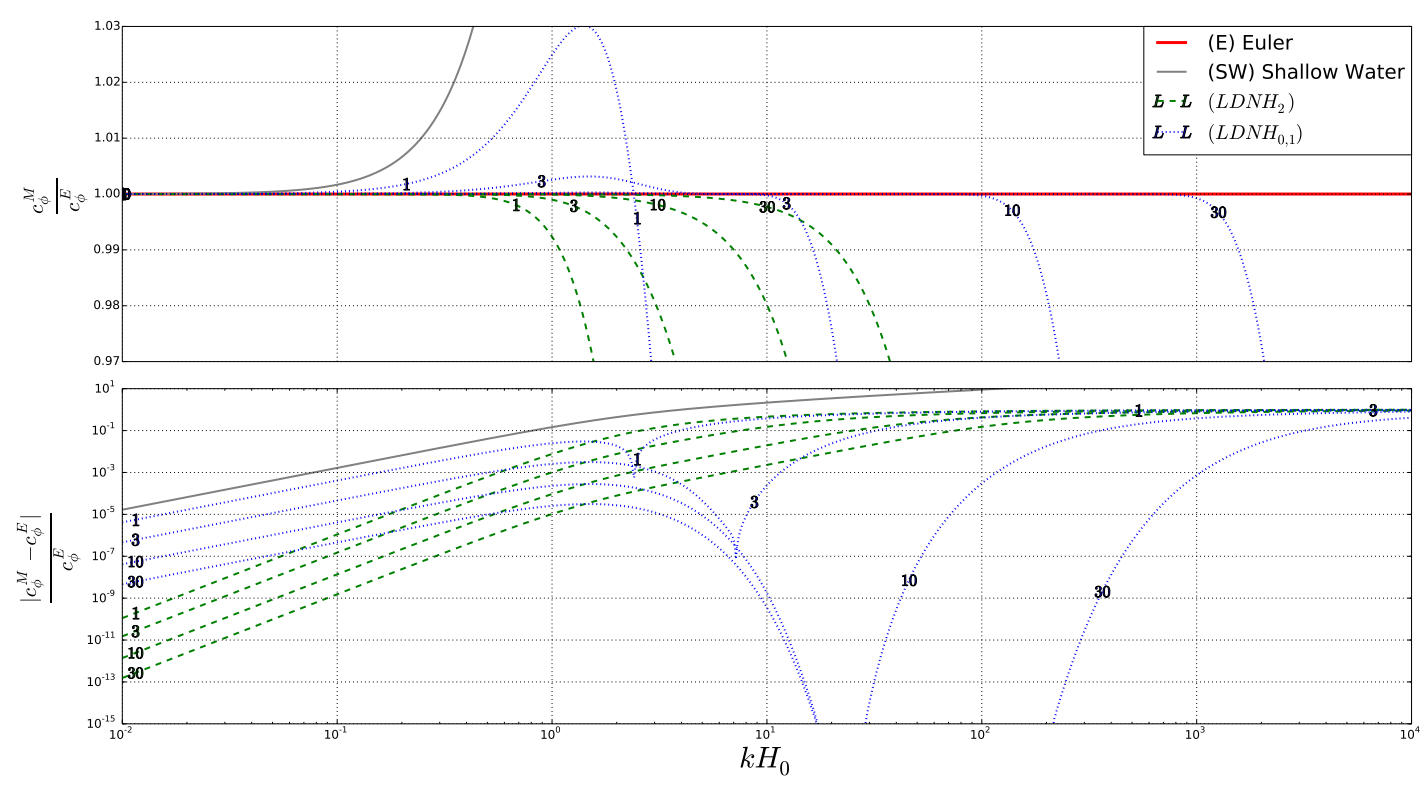

Figure 2: Dispersion relations for Euler (red thick solid line), Shallow Water (gray thin solid line), $\mathrm{LDNH}_{2}$ model (green dashed line) and $\mathrm{LDNH}_{0} / \mathrm{LDNH}_{1}$ models (blue dotted line)

Remark 9 Ratios (4.29) can be interpreted as Padé's approximants for the exact celerity (4.30). Previous works (e.g. [6,39]) are based on such approximants that guided the procedure to incorporate new terms into the model in order to improve the accuracy of the approximation.

\subsubsection{Comparison of dispersion relations}

We observe on Figure 2 the celerity associated to the Euler equations, the shallow water equations as well as $\mathrm{LDNH}_{2}$ and $\mathrm{LDNH}_{0}$ models for $L$ layers $(L \in\{1,3,10,30\})$. In accordance with the literature, the shallow water equations are more legitimate for large wavelengths (small wave numbers $k$ ).

As expected, the larger $L$, the more accurate the multilayer models. Multiplying the number of layers by 3 induces a gain of one order of magnitude of the error. For a given magnitude of error, we can thus choose a relevant number of layers depending on the range of wave numbers for the associated physical phenomenon.

It is difficult to discriminate the $\mathrm{LDNH}_{2}$ and the $\mathrm{LDNH}_{0}$ models since they have distinct monotonicities. For small wave numbers, the $\mathrm{LDNH}_{2}$ model looks more accurate while for large wave numbers the $\mathrm{LDNH}_{0}$ model seems to provide better results. Anyway, as stated in Theorem 1, dispersion relations converge to the one of the Euler equations.

Linear dispersion relations are usually exhibited in the literature to assess dispersive models $[28,39,43,44]$. In the latter references, linear relations for (monolayer) Boussinesq type models are provided with parameters tuned to fit Airy's formula for $k H_{0}$ as large as possible. Those are recalled and pictured for instance in [35, Fig. 1] and [6, Fig. 3]. As for the present models, all are accurate for shallow water flows but became irrelevant for deep waters.

This raises the following modelling issue: either taking into account higher order derivatives like in BTM models or increasing the number of layers as in the LDNH framework. We chose here to focus on nonhydrostatic models where low order derivatives are involved and satisfying energy estimates. Figure 2 shows that accuracy is strongly improved with an increasing number of layers but this will have to be analysed in future works with regard to the computational cost to find some adequate balance. 


\section{Design of the hierarchy}

We shall specify in this last section the link between the three models $\left(\mathrm{LDNH}_{\nu}\right)_{\nu \in\{0,1,2\}}$ derived in this paper. More precisely we shall underline how they are related to each other within the same hierarchy and how they can be distinguished.

First of all, let us mention that the three of them have a common core made of conservation laws for water volume (3.9a) and momentum (3.9b-3.9c). Discrepancies arise for other equations especially those characterising the hydrodynamic component of the pressure field. Indeed, interfacial and mean values of the hydrodynamic pressure in $\mathrm{LDNH}_{2}$ are related by Eq. (3.9d) which can be written

$$
\begin{aligned}
q_{\alpha}=\frac{q_{\alpha+1 / 2}+q_{\alpha-1 / 2}}{2}+\frac{1}{2 \sqrt{3}}\left(\partial_{t}\left(h_{\alpha} \sigma_{\alpha}\right)+\partial_{x}\left(h_{\alpha} \sigma_{\alpha} u_{\alpha}\right)\right)+\Gamma_{\alpha+1 / 2}\left(\frac{h_{\alpha} \partial_{x} u_{\alpha}}{12}+\frac{\widetilde{w}_{\alpha+1 / 2}-w_{\alpha}}{2}\right) & \\
& -\Gamma_{\alpha-1 / 2}\left(\frac{h_{\alpha} \partial_{x} u_{\alpha}}{12}+\frac{w_{\alpha}-\widetilde{w}_{\alpha-1 / 2}}{2}\right) .
\end{aligned}
$$

Its counterpart in $\mathrm{LDNH}_{1}$ - namely (3.14) - comprises fewer differential terms accounting for the lower order of approximation. Finally in $\mathrm{LDNH}_{0}$, it reduces to the simple average (3.15b).

To go further, let us focus on the very difference between $\mathrm{LDNH}_{2}$ and $\mathrm{LDNH}_{0}$ by considering the approximation of each unknown. The $\mathrm{LDNH}_{2}$-model corresponds to a $\left(\mathbb{P}_{0}, \mathbb{P}_{1}, \mathbb{P}_{2}\right)$-discretisation for $(u, w, q)$ while it is $\left(\mathbb{P}_{0}, \mathbb{P}_{0}, \mathbb{P}_{1}\right)$ for the $\mathrm{LDNH}_{0}$-model. This implies more degrees of freedom in the $\mathrm{LDNH}_{2}$-model and explains why the kinetic energy is expressed differently - compare (4.15) and (4.20). Despite these different expressions, both systems satisfy an energy inequality (Prop. 1) which transcribes the fact that models are dissipative. Likewise, both systems have different linear dispersion relations (Th. 1) which make them more accurate (in accordance with Airy's formula) in different ranges of wave numbers.

Model $\mathrm{LDNH}_{1}$ is an intermediate system between $\mathrm{LDNH}_{2}$ and $\mathrm{LDNH}_{0}$ insofar as it shares properties with both of them. First of all, primary variables $(u, w, q)$ are discretised the same way as for $\mathrm{LDNH}_{2}$, namely in a $\left(\mathbb{P}_{0}, \mathbb{P}_{1}, \mathbb{P}_{2}\right)$ approximation but the truncation made in $\mathrm{LDNH}_{1}$ amounts to assuming the energy variable in $\mathbb{P}_{0}$ rather than in $\mathbb{P}_{2}$. That is why we consider the same kinetic energy $\bar{K}_{\alpha}$ as in $\mathrm{LDNH}_{0}$ rather than $\overline{\mathcal{K}}_{\alpha}$ as in $\mathrm{LDNH}_{2}$. The choice for $\gamma_{\alpha+1 / 2}$ induces an exact energy equality (Prop. 3). Second of all, the linear dispersion relation satisfied by $\mathrm{LDNH}_{1}$ is the same as $\mathrm{LDNH}_{0}$ as mass transfer terms (which are the very difference between $\mathrm{LDNH}_{1}$ and $\mathrm{LDNH}_{0}$ ) vanish in the linearisation procedure.

\section{Conclusion}

A hierarchy of layerwise discretisation models with hydrodynamic pressure has been proposed in this paper. They can be seen as a splitting method which improves the vertical accuracy of NLSW equations. The number of layers defines the vertical partition of the moving domain, whose bottom and free surface can vary in time and to include wet/dry fronts. When the number of layers is set to 1 , the proposed models $\mathrm{LDNH}_{0}$ and $\mathrm{LDNH}_{1}$ coincide with the model proposed in [2] (which can also seen as a generalisation of the model proposed in [55]) while model $\mathrm{LDNH}_{2}$ with a single layer coincides with the Serre-Green-Naghdi model [29, $37,50,53]$.

The hierarchy of models corresponds to the level of vertical accuracy that is considered during the derivation. In all cases the only requirement that we considered is to obtain a model with a hydrodynamic pressure and verifying an exact energy balance. In particular, the horizontal velocity has a constant vertical profile within each layer and a linear vertical profile of the vertical velocity is considered in the discretisation of the incompressibility condition equation. The differences between the three models appear in the hypotheses upon the vertical velocity in the discretisation of the vertical momentum equation and upon the pressure profile.

In addition to energy estimates, the main result of the paper is an explicit formula for the dispersion relation for an arbitrary number of layers no matter what the models in the hierarchy. As a consequence, we notice 
that when the number of layers increases, the celerity converges to the celerity associated to the Euler equations.

In a forthcoming paper the authors will address the numerical approximation of the three proposed models, including comparisons with experimental data and comparisons of each model with each other from an efficiency point of view. Moreover, only the Euler equations were considered in the present paper. Another field of investigation consists in extending this approach to the approximation of the Navier-Stokes equations by taking into account viscous terms as it was studied in the hydrostatic case in [14].

\section{Acknowledgments}

The authors do thank Emmanuel Audusse (Univ. Paris 13) and Tomas Morales de Luna (Univ. Córdoba) for fruitful discussions about multilayer models. Y. Penel is grateful to IMUS (Univ. Sevilla) and Inria Project Lab. Algae in Silico for partially funding a 3-month stay at Sevilla. This research has been partially supported by the Spanish Government and FEDER through the research project MTM2015-70490-C2-2-R.

\section{A Dispersion relation}

\section{A.1 Proof of Lemma 1}

$\mathrm{LDNH}_{2}$ case. Inserting the plane wave form into (4.26) provides

$$
\left\{\begin{array}{l}
\omega \hat{H}=k H_{0} \sum_{\alpha=1}^{L} \ell_{\alpha} \hat{u}_{\alpha} \\
\omega \hat{u}_{\alpha}=k \hat{q}_{\alpha}+k g \hat{H} \\
-i \omega \ell_{\alpha} H_{0} \hat{w}_{\alpha}+\hat{q}_{\alpha+1 / 2}-\hat{q}_{\alpha-1 / 2}=0 \\
\omega k \ell_{\alpha}^{2} H_{0}^{2} \hat{u}_{\alpha}+12\left[\hat{q}_{\alpha}-\frac{\hat{q}_{\alpha+1 / 2}+\hat{q}_{\alpha-1 / 2}}{2}\right]=0 \\
\hat{w}_{\alpha}=-i k H_{0}\left[\frac{\ell_{\alpha}}{2} \hat{u}_{\alpha}+\sum_{\beta=1}^{\alpha-1} \ell_{\beta} \hat{u}_{\beta}\right]
\end{array}\right.
$$

Summing (A.1c) from $L$ to $\alpha+1$ yields given the boundary condition $\hat{q}_{L+1 / 2}=0$

$$
\hat{q}_{\alpha+1 / 2}=-i \omega H_{0} \sum_{\beta=\alpha+1}^{L} \ell_{\beta} \hat{w}_{\beta} .
$$

Equality (A.1d) then reads

$$
\begin{aligned}
\hat{q}_{\alpha}= & -\frac{\omega k \ell_{\alpha}^{2} H_{0}^{2}}{12} \hat{u}_{\alpha}-i \omega H_{0}\left[\sum_{\beta=\alpha+1}^{L} \ell_{\beta} \hat{w}_{\beta}+\frac{\ell_{\alpha}}{2} \hat{w}_{\alpha}\right] \\
& \stackrel{(\text { A.1e) }}{=}-\frac{\omega k \ell_{\alpha}^{2} H_{0}^{2}}{12} \hat{u}_{\alpha}-\omega k H_{0}^{2}\left[\sum_{\beta=\alpha+1}^{L} \frac{\ell_{\beta}^{2}}{2} \hat{u}_{\beta}+\sum_{\beta=1}^{L}\left(\sum_{\gamma=\max \{\alpha+1, \beta+1\}}^{L} \ell_{\gamma}\right) \ell_{\beta} \hat{u}_{\beta}+\frac{\ell_{\alpha}^{2}}{4} \hat{u}_{\alpha}+\frac{\ell_{\alpha}}{2} \sum_{\beta=1}^{\alpha-1} \ell_{\beta} \hat{u}_{\beta}\right] .
\end{aligned}
$$

Finally (A.1b) becomes

$$
\omega^{2} \hat{u}_{\alpha}-k^{2} g H_{0} \sum_{\beta=1}^{L} \ell_{\beta} \hat{u}_{\beta}+\omega^{2} k^{2} H_{0}^{2}\left[\frac{\ell_{\alpha}^{2}}{3} \hat{u}_{\alpha}+\sum_{\beta=\alpha+1}^{L} \frac{\ell_{\beta}^{2}}{2} \hat{u}_{\beta}+\sum_{\beta=1}^{L}\left(\sum_{\gamma=\max \{\alpha, \beta\}+1}^{L} \ell_{\gamma}\right) \ell_{\beta} \hat{u}_{\beta}+\frac{\ell_{\alpha}}{2} \sum_{\beta=1}^{\alpha-1} \ell_{\beta} \hat{u}_{\beta}\right]=0 .
$$


The $L$ equations for $\hat{\boldsymbol{u}}=\left(\hat{u}_{\alpha}\right)_{1 \leq \alpha \leq L}$ form a homogeneous linear system $\widetilde{\mathcal{A}} \hat{\boldsymbol{u}}=0$ where $\widetilde{\mathcal{A}}(\omega, k)=\omega^{2} \mathcal{A}_{k H_{0}}-$ $k^{2} g H_{0} \boldsymbol{e} \otimes \boldsymbol{\ell}$ and $\mathcal{A}$ defined by (4.28).

The existence of a non-trivial solution to $\widetilde{\mathcal{A}} \hat{\boldsymbol{u}}=0$ requires that $\operatorname{det} \widetilde{\mathcal{A}}(\omega, k)=0$ which provides the dispersion relation. It can also be recovered by noticing that $\widetilde{\mathcal{A}} \hat{\boldsymbol{u}}=0$ is equivalent to $\omega^{2} \mathcal{A}_{k H_{0}} \hat{\boldsymbol{u}}=k^{2} g H_{0}\langle\hat{\boldsymbol{u}}, \boldsymbol{\ell}\rangle \boldsymbol{e}$. Provided that $\mathcal{A}_{k H_{0}}$ is invertible, then necessarily $\langle\hat{\boldsymbol{u}}, \boldsymbol{\ell}\rangle \neq 0$ and we recover (4.27) for $\lambda=3$.

$\mathrm{LDNH}_{1,0}$ case. Insofar as terms involving $\Gamma_{\alpha+1 / 2}$ disappear in the linearising process, Models $\mathrm{LDNH}_{1}$ and $\mathrm{LDNH}_{0}$ share the same linearised formulation. They admit a plane wave solution if the following equations are satisfied

$$
\left\{\begin{array}{l}
\omega \hat{H}=k H_{0} \sum_{\alpha=1}^{L} \ell_{\alpha} \hat{u}_{\alpha}, \\
\omega \hat{u}_{\alpha}=k \hat{q}_{\alpha}+k g \hat{H}, \\
-i \omega \ell_{\alpha} H_{0} \hat{w}_{\alpha}+\hat{q}_{\alpha+1 / 2}-\hat{q}_{\alpha-1 / 2}=0, \\
\hat{q}_{\alpha}=\frac{\hat{q}_{\alpha+1 / 2}+\hat{q}_{\alpha-1 / 2}}{2}, \\
\hat{w}_{\alpha}=-i k H_{0}\left[\frac{\ell_{\alpha}}{2} \hat{u}_{\alpha}+\sum_{\beta=1}^{\alpha-1} \ell_{\beta} \hat{u}_{\beta}\right] .
\end{array}\right.
$$

Similarly to the $\mathrm{LDNH}_{2}$ case, pressure terms can be expressed by

$$
\begin{gathered}
\hat{q}_{\alpha+1 / 2}=-i \omega H_{0} \sum_{\beta=\alpha+1}^{L} \ell_{\beta} \hat{w}_{\beta}, \\
\hat{q}_{\alpha}=-\omega k H_{0}^{2}\left[\sum_{\beta=\alpha+1}^{L} \frac{\ell_{\beta}^{2}}{2} \hat{u}_{\beta}+\sum_{\beta=1}^{L}\left(\sum_{\gamma=\max \{\alpha+1, \beta+1\}}^{L} \ell_{\gamma}\right) \ell_{\beta} \hat{u}_{\beta}+\frac{\ell_{\alpha}^{2}}{4} \hat{u}_{\alpha}+\frac{\ell_{\alpha}}{2} \sum_{\beta=1}^{\alpha-1} \ell_{\beta} \hat{u}_{\beta}\right] .
\end{gathered}
$$

Finally (A.2b) becomes

$$
\omega^{2} \hat{u}_{\alpha}-k^{2} g H_{0} \sum_{\beta=1}^{L} \ell_{\beta} \hat{u}_{\beta}+\omega^{2} k^{2} H_{0}^{2}\left[\frac{\ell_{\alpha}^{2}}{4} \hat{u}_{\alpha}+\sum_{\beta=\alpha+1}^{L} \frac{\ell_{\beta}^{2}}{2} \hat{u}_{\beta}+\sum_{\beta=1}^{L}\left(\sum_{\gamma=\max \{\alpha, \beta\}+1}^{L} \ell_{\gamma}\right) \ell_{\beta} \hat{u}_{\beta}+\frac{\ell_{\alpha}}{2} \sum_{\beta=1}^{\alpha-1} \ell_{\beta} \hat{u}_{\beta}\right]=0 .
$$

The existence of a non-trivial solution to this homogeneous linear system leads to the dispersion relation (4.27) for $\lambda=2$.

\section{A.2 Proof of Theorem 1}

We focus on the homogeneous case. Let us introduce the symmetric matrix

$$
\widetilde{\mathcal{B}}(x, y)=\mathcal{I}_{L}+x^{2} \mathcal{B}-\frac{y^{2}}{L} \boldsymbol{e} \otimes \boldsymbol{e},
$$

where $\mathcal{B}$ is defined by (4.28), which reduces to

$$
\mathcal{B}_{\alpha \beta}=\frac{1}{L^{2}}\left(-\frac{\delta_{\alpha \beta}}{6}+\frac{1}{2}+L-\max \{\alpha, \beta\}\right)
$$

$\widetilde{\mathcal{B}}$ is then such that

$$
\frac{1}{\omega^{2}} \widetilde{\mathcal{A}}(\omega, k)=\widetilde{\mathcal{B}}(x, y) \quad \text { for } \quad x=k H_{0}, y=\frac{k \sqrt{g H_{0}}}{\omega} .
$$


The existence of a non-trivial vector $\hat{\boldsymbol{u}} \neq 0$ such that $\widetilde{\mathcal{A}} \hat{\boldsymbol{u}}=0$ is equivalent to showing that $\operatorname{det} \widetilde{\mathcal{B}}(x, y)=0$.

Let us also set

$$
\chi=1-\frac{x^{2}}{2 \lambda L^{2}}, \quad \psi=-2\left(1+\frac{\lambda-1}{2 \lambda} \frac{x^{2}}{L^{2}}\right), \quad \text { and } \quad \zeta=-3+\frac{3-2 \lambda}{2 \lambda} \frac{x^{2}}{L^{2}} .
$$

The following procedures ${ }^{7}$

$$
\begin{aligned}
& \text { 1: for } i=2 \text { to } L-1 \text { do } \\
& \text { 2: } \quad R_{i} \leftarrow R_{i}-R_{1} \\
& \text { 3: for } j=1 \text { to } L-2 \text { do } \\
& \text { 4: } \quad C_{j} \leftarrow C_{j}-2 C_{j+1}+C_{j+2} \\
& \text { 5: } C_{L-1} \leftarrow C_{L-1}-\kappa C_{L} \text { for a suitable } \kappa \text { such that the first coefficient vanishes }
\end{aligned}
$$

lead to $\operatorname{det} \widetilde{\mathcal{B}}=\operatorname{det} \mathcal{D}$, where $\mathcal{D}=\mathcal{T}+\tau E_{1 L}$ (see the profile on Fig. 3 and (A.3) for notations) and

- $\tau=\frac{x^{2}}{2 L^{2}}-\frac{y^{2}}{L}$

- $\mathcal{T}$ is a sparse lower triangular matrix whose non-zero coefficients are:

$\star \mathcal{T}_{i, i}=\chi$ for $i \in\{1, \ldots, L-1\}$ and $\mathcal{T}_{L, L}=-\frac{\psi}{2}-\frac{y^{2}}{L}$

$\star \mathcal{T}_{2,1}=\zeta, \mathcal{T}_{i, i-1}=\psi$ for $i \in\{3, \ldots, L-1\}$,

$$
\mathcal{T}_{L, L-1}=-\left(1+\frac{2 \lambda-1}{2 \lambda} \frac{x^{2}}{L^{2}}\right)-\frac{\chi \frac{x^{2}}{L^{2}}}{\frac{x^{2}}{2 L^{2}}-\frac{y^{2}}{L}} ;
$$

$\star \mathcal{T}_{3,1}=0, \mathcal{T}_{i, i-2}=\chi$ for $i \in\{4, \ldots, L\}$

$\star \mathcal{T}_{i, 1}=-\chi$ for $i \in\{4, \ldots, L-1\}, \mathcal{T}_{L, 1}=0$.

A cofactor expansion (with respect to the last column) of $\operatorname{det} \mathcal{D}$ yields

$$
\operatorname{det} \mathcal{D}=\prod_{i=1}^{L} \mathcal{T}_{i, i}+(-1)^{L+1} \tau \operatorname{det} \mathcal{J}
$$

where $\mathcal{J}$ is the sub-matrix extracted from $\mathcal{T}$ (rows 2 to $L$ and columns 1 to $L-1$ ). Its determinant is computed by means of a descent algorithm to keep a single non-zero coefficient in the first column.

$$
\mid \begin{aligned}
& \text { 1: for } k=1 \text { to } L-2 \text { do } \\
& 2: \quad \boldsymbol{f}^{(k)}=\boldsymbol{f}^{(k-1)}-\frac{f_{k}^{(k-1)}}{\mathcal{J}_{k, k+1}} \boldsymbol{j}_{k+1}
\end{aligned}
$$

where $\boldsymbol{f}^{(0)}=\boldsymbol{j}_{1}$ and $\boldsymbol{j}_{k}$ is the $k^{\text {th }}$ column vector of $\mathcal{J}$, i.e.

$$
\boldsymbol{j}_{1}=\left(\begin{array}{c}
\zeta \\
0 \\
-\chi \\
\vdots \\
-\chi \\
0
\end{array}\right), \boldsymbol{j}_{k}=\left(\begin{array}{c}
0 \\
\chi \\
\psi \\
\chi \\
0
\end{array}\right) \leftarrow k \text { for } k \in\{2, \ldots, L-2\}, \boldsymbol{j}_{L-1}=\left(\begin{array}{c}
0 \\
\vdots \\
0 \\
\chi \\
\mathcal{T}_{L, L-1}
\end{array}\right)
$$

\footnotetext{
${ }^{7} R_{i}$ and $C_{j}$ denote respectively the $i^{\text {th }}$ row and the $j^{\text {th }}$ column of the matrix.
} 
Finally, vector $\boldsymbol{f}^{(L-2)}$ is proportional to $\boldsymbol{e}_{L-1}$ and can be computed explicitly. Indeed, introducing sequences $\left(\mathfrak{a}_{k}=f_{k+1}^{(k)}\right)$ and $\left(\mathfrak{b}_{k}=f_{k+2}^{(k)}\right)$, i.e. such that

$$
\boldsymbol{f}^{(k)}=\left(\begin{array}{c}
0 \\
\vdots \\
0 \\
\mathfrak{a}_{k} \\
\mathfrak{b}_{k} \\
\star \\
\star
\end{array}\right) \leftarrow k+1,
$$

where $\star$ are original coefficients of $\boldsymbol{j}_{1}$, then we have

(1) $\mathfrak{a}_{0}=\zeta$ and $\forall k \in\{1, \ldots, L-3\}, \mathfrak{a}_{k}=\mathfrak{b}_{k-1}+2 \gamma \mathfrak{a}_{k-1}$ with $\gamma=\frac{-\psi}{2 \chi}$;

(2) $\mathfrak{a}_{L-2}=\mathfrak{b}_{L-3}-\frac{\mathcal{T}_{L, L-1}}{\chi} \mathfrak{a}_{L-3}$

(3) $\mathfrak{b}_{0}=0$ and $\forall k \in\{1, \ldots, L-4\}, \mathfrak{b}_{k}=-\chi-\mathfrak{a}_{k-1}$;

(4) $\mathfrak{b}_{L-3}=-\mathfrak{a}_{L-4}$.

(1) corresponds to the $(k+1)^{\text {th }}$ row of Step $k \in\{1, \ldots, L-3\}$ in the algorithm above, (2) for $k=L-2$, (3) to the $(k+2)^{\text {th }}$ row for Step $k \in\{1, \ldots, L-4\}$ and (4) for $k=L-3$.

Hence, for all $k \in\{0, \ldots, L-5\}$, the sequence satisfies the second-order linear induction relation

$$
\mathfrak{a}_{k+2}-2 \gamma \mathfrak{a}_{k+1}+\mathfrak{a}_{k}=-\chi .
$$

Let us set $\widetilde{\mathfrak{a}}_{k}=\mathfrak{a}_{k}+\frac{\chi}{2(1-\gamma)}$ such that $\left(\widetilde{\mathfrak{a}}_{k}\right)$ is the solution of the homogeneous counterpart of (A.4). We also introduce

$$
\eta=1+\frac{\lambda-1}{2 \lambda} \frac{x^{2}}{L^{2}}, \delta=\frac{x^{2}}{L^{2}}\left(1+\frac{\lambda-2}{4 \lambda} \frac{x^{2}}{L^{2}}\right), r_{ \pm}=\eta \pm \sqrt{\delta} \text { and } \theta=\frac{\widetilde{\mathfrak{a}}_{0} r_{-}-\widetilde{\mathfrak{a}}_{1} \chi}{r_{-}-r_{+}} .
$$

Then the solution to (A.4) satisfies

$$
\chi^{k} \mathfrak{a}_{k}=\theta r_{+}^{k}+\left(\widetilde{\mathfrak{a}}_{0}-\theta\right) r_{-}^{k}+\frac{L^{2}}{x^{2}} \chi^{k+2} .
$$

By means of a cofactor expansion (with respect to the first column whose coefficients are 0 except the last one) of $\operatorname{det} \mathcal{J}$, we finally obtain

$$
\begin{aligned}
\operatorname{det} \mathcal{D} & =\prod_{i=1}^{L} \mathcal{T}_{i, i}+(-1)^{L+1} \tau \times(-1)^{L} \mathfrak{a}_{L-2} \prod_{i=2}^{L-1} \mathcal{T}_{i, i} \\
& =\chi^{L-2}\left[\chi\left(1+\frac{\lambda-1}{2 \lambda} \frac{x^{2}}{L^{2}}-\frac{y^{2}}{L}\right)-\mathfrak{a}_{L-2}\left(\frac{x^{2}}{2 L^{2}}-\frac{y^{2}}{L}\right)\right] .
\end{aligned}
$$

We mention that $\mathfrak{a}_{k}$ is independent from $y$ for $k \in\{0, \ldots, L-3\}$ but $\mathfrak{a}_{L-2}=-\mathfrak{a}_{L-4}-\frac{\mathcal{T}_{L, L-1}}{\chi} \mathfrak{a}_{L-3}$ does as $\mathcal{T}_{L, L-1}$ is a function of $y$. Hence, we isolate $y$ in the equation $\operatorname{det} \mathcal{D}=0$ which provides the following relation

$$
\frac{L}{y^{2}}=\frac{\chi^{L-1}+\chi^{2}\left\{\chi^{L-4} \mathfrak{a}_{L-4}\right\}-\left(1+\frac{2 \lambda-1}{2 \lambda} \frac{x^{2}}{L^{2}}\right)\left\{\chi^{L-3} \mathfrak{a}_{L-3}\right\}}{\chi^{L-1}\left(1+\frac{\lambda-1}{2 \lambda} \frac{x^{2}}{L^{2}}\right)+\chi^{2} \frac{x^{2}}{2 L^{2}}\left\{\chi^{L-4} \mathfrak{a}_{L-4}\right\}-\frac{x^{2}}{2 L^{2}}\left(3+\frac{2 \lambda-3}{2 \lambda} \frac{x^{2}}{L^{2}}\right)\left\{\chi^{L-3} \mathfrak{a}_{L-3}\right\}} .
$$

Expressions $\chi^{k} \mathfrak{a}_{k}$ between braces are computed thanks to (A.6).

We can infer the limit of $y^{2}$ as $L \rightarrow+\infty$. Indeed, Taylor expansions with respect to $L^{-1}$ read 
- $r_{ \pm} \stackrel{(\text { A.5) }}{=} 1 \pm \frac{x}{L}+\mathcal{O}\left(L^{-2}\right) \quad \Longrightarrow \quad r_{ \pm}^{L}=\exp ( \pm x)+\mathcal{O}\left(L^{-1}\right)$;

- $\tilde{\mathfrak{a}}_{0}=-\frac{L^{2}}{x^{2}}\left(1+\frac{3 \lambda-1}{\lambda} \frac{x^{2}}{L^{2}}+\frac{1-6 \lambda+4 \lambda^{2}}{4 \lambda^{2}} \frac{x^{4}}{L^{4}}\right)=-\frac{L^{2}}{x^{2}}+\mathcal{O}(1) \quad \Longrightarrow \quad \tilde{\mathfrak{a}}_{0} r_{-}=-\frac{L^{2}}{x^{2}}+\frac{L}{x}+\mathcal{O}(1)$;

- $\tilde{\mathfrak{a}}_{1}=-\frac{1}{\chi}\left[\frac{L^{2}}{x^{2}} \chi^{3}+\psi \zeta\right] \quad \Longrightarrow \quad \tilde{\mathfrak{a}}_{1} \chi=-\frac{L^{2}}{x^{2}}+\mathcal{O}(1)$

- $r_{-}-r_{+}=-\frac{2 x}{L}+\mathcal{O}\left(L^{-3}\right) \quad \Longrightarrow \quad \theta \stackrel{(\text { A.5) }}{=}-\frac{L^{2}}{2 x^{2}}+\mathcal{O}(L)$.

We deduce that the numerator in (A.7) verifies

$$
\begin{aligned}
\chi^{L-1}+ & \chi^{2}\left(\theta r_{+}^{L-4}+\left(\widetilde{\mathfrak{a}}_{0}-\theta\right) r_{-}^{L-4}+\frac{L^{2}}{x^{2}} \chi^{L-2}\right)-\left(1+\frac{2 \lambda-1}{2 \lambda} \frac{x^{2}}{L^{2}}\right)\left(\theta r_{+}^{L-3}+\left(\widetilde{\mathfrak{a}}_{0}-\theta\right) r_{-}^{L-3}+\frac{L^{2}}{x^{2}} \chi^{L-1}\right) \\
= & \frac{\theta}{r_{+}^{4}} r_{+}^{L}\left[\chi^{2}-\left(1+\frac{2 \lambda-1}{2 \lambda} \frac{x^{2}}{L^{2}}\right) r_{+}\right]+\frac{\widetilde{\mathfrak{a}}_{0}-\theta}{r_{-}^{4}} r_{-}^{L}\left[\chi^{2}-\left(1+\frac{2 \lambda-1}{2 \lambda} \frac{x^{2}}{L^{2}}\right) r_{-}\right] \\
& +\frac{L^{2}}{x^{2}} \chi^{L-1}\left[\frac{x^{2}}{L^{2}}+\chi-\left(1+\frac{2 \lambda-1}{2 \lambda} \frac{x^{2}}{L^{2}}\right)\right] \\
= & \frac{L}{x} \sinh x+\mathcal{O}(1) .
\end{aligned}
$$

Likewise, the denominator in (A.7) satisfies

$$
\begin{aligned}
\chi^{L-1}\left(1+\frac{\lambda-1}{2 \lambda} \frac{x^{2}}{L^{2}}\right) & +\frac{x^{2} \chi^{2}}{2 L^{2}}\left(\theta r_{+}^{L-4}+\left(\widetilde{\mathfrak{a}}_{0}-\theta\right) r_{-}^{L-4}+\frac{L^{2}}{x^{2}} \chi^{L-2}\right) \\
& -\frac{x^{2}}{2 L^{2}}\left(3+\frac{2 \lambda-3}{2 \lambda} \frac{x^{2}}{L^{2}}\right)\left(\theta r_{+}^{L-3}+\left(\widetilde{\mathfrak{a}}_{0}-\theta\right) r_{-}^{L-3}+\frac{L^{2}}{x^{2}} \chi^{L-1}\right)=\cosh x+\mathcal{O}\left(L^{-1}\right) .
\end{aligned}
$$

Combining the two last results shows that (A.7) implies

$$
\frac{1}{y^{2}} \underset{L \rightarrow+\infty}{\longrightarrow} \frac{\tanh x}{x} \text {. }
$$

To conclude, we notice that given (A.5), (A.6) expands as

$$
\begin{aligned}
\chi^{k}\left(\mathfrak{a}_{k}-\frac{L^{2} \chi^{2}}{x^{2}}\right) & =\sum_{j=0}^{k}\left(\begin{array}{c}
k \\
j
\end{array}\right) \eta^{k-j} \delta^{j / 2}\left[\theta+(-1)^{j}\left(\widetilde{\mathfrak{a}}_{0}-\theta\right)\right] \\
& =\tilde{\mathfrak{a}}_{0} \sum_{0 \leq 2 m \leq k}\left(\begin{array}{c}
k \\
2 m
\end{array}\right) \eta^{k-2 m} \delta^{m}+\sqrt{\delta}\left(2 \theta-\widetilde{\mathfrak{a}}_{0}\right) \sum_{0 \leq 2 m+1 \leq k}\left(\begin{array}{c}
k \\
2 m+1
\end{array}\right) \eta^{k-2 m-1} \delta^{m} \\
& =\tilde{\mathfrak{a}}_{0} \sum_{0 \leq 2 m \leq k}\left(\begin{array}{c}
k \\
2 m
\end{array}\right) \eta^{k-2 m} \delta^{m}+\left(\chi \widetilde{\mathfrak{a}}_{1}-\eta \widetilde{\mathfrak{a}}_{0}\right) \sum_{0 \leq 2 m+1 \leq k}\left(\begin{array}{c}
k \\
2 m+1
\end{array}\right) \eta^{k-2 m-1} \delta^{m}
\end{aligned}
$$

which shows that no square root is involved. As $\chi \widetilde{\mathfrak{a}}_{1}-\eta \widetilde{\mathfrak{a}}_{0}=-\frac{5}{2}+\frac{5(1-\lambda)}{2 \lambda} \frac{x^{2}}{L^{2}}+\frac{-\frac{5}{2}+5 \lambda-2 \lambda^{2}}{4 \lambda^{2}} \frac{x^{4}}{L^{4}}$, we obtain (4.29).

\section{B Some properties of the $\mathrm{LDNH}_{1}$ model}

\section{B.1 Derivation of $\mathrm{LDNH}_{1}$}

This paragraph is restricted to the case of homogeneous layers: $h_{\alpha}=\frac{H}{L}, \alpha \in\{1, \ldots, L\}$. As explained in $\S 4.1 .2$, we have to deal with integrals of quadratic polynomials and we used in the $\mathrm{LDNH}_{2}$ case exact quadrature formulae. If we rather use a 1st-order Hermitte interpolation polynomial, i.e.

$$
z \underline{w}_{\mid \mathcal{L}_{\alpha}} \approx z_{\alpha} w_{\alpha}+\left(z-z_{\alpha}\right)\left(w_{\alpha}-z_{\alpha} \partial_{x} u_{\alpha}\right) \quad \text { and } \quad \underline{w}_{\mid \mathcal{L}_{\alpha}}^{2} \approx w_{\alpha}^{2}-2\left(z-z_{\alpha}\right) w_{\alpha} \partial_{x} u_{\alpha},
$$


the application of $(4.2 \mathrm{a})$ to $(4.11)$ yields

$$
\begin{aligned}
\partial_{t}\left(h_{\alpha} z_{\alpha} w_{\alpha}\right)+\partial_{x}\left(h_{\alpha} z_{\alpha} u_{\alpha} w_{\alpha}\right)+\Gamma_{\alpha+1 / 2}\left(z_{\alpha+1 / 2} \widetilde{w}_{\alpha+1 / 2}+\frac{H^{2}}{4 L^{2}} \widetilde{\left(\partial_{x} u\right)_{\alpha+1 / 2}}\right)+z_{\alpha+1 / 2} q_{\alpha+1 / 2} \\
-\Gamma_{\alpha-1 / 2}\left(z_{\alpha-1 / 2} \widetilde{w}_{\alpha-1 / 2}+\frac{H^{2}}{4 L^{2}} \widetilde{\left(\partial_{x} u\right)_{\alpha-1 / 2}}\right)-z_{\alpha-1 / 2} q_{\alpha-1 / 2}=h_{\alpha}\left(w_{\alpha}^{2}+q_{\alpha}\right) .
\end{aligned}
$$

We then verify similarly to the $\mathrm{LDNH}_{2}$ case that smooth solutions to (B.1) are also solutions to (3.14). Notice that the Hermitte interpolation process makes sense for $z$ in the vicinity of $z_{\alpha}$, which holds when $L \gg 1$.

\section{B.2 Energy}

In accordance with the approximation made above, the kinetic energy for the $\mathrm{LDNH}_{1}$-model reduces to (4.20) replacing the corresponding kinetic energy (4.15) for the $\mathrm{LDNH}_{2}$-model. The result reads:

\section{Proposition 3}

Let us take $\gamma_{\alpha+1 / 2}=\frac{1}{2}$. If $\left(u_{\alpha}, w_{\alpha}, q_{\alpha}\right)$ are smooth solutions to $\mathrm{LDNH}_{1}$, we have

$$
\begin{aligned}
\partial_{t}\left(\sum_{\alpha=1}^{L} h_{\alpha}\left(\bar{K}_{\alpha}+g z_{\alpha}+p^{a t m}\right)\right)+\partial_{x}\left(\sum _ { \alpha = 1 } ^ { L } h _ { \alpha } u _ { \alpha } \left(\bar{K}_{\alpha}+q_{\alpha}+g \eta\right.\right. & \left.\left.+p^{a t m}\right)\right) \\
& =H \partial_{t} p^{a t m}+\left(g H+q_{1 / 2}\right) \partial_{t} z_{b} .
\end{aligned}
$$

Proof: The proof is quite similar to that of Prop. 1. We have

$$
\begin{aligned}
\partial_{t}\left(h_{\alpha} \bar{K}_{\alpha}\right)+ & \partial_{x}\left(h_{\alpha} u_{\alpha}\left[\bar{K}_{\alpha}+q_{\alpha}+g \eta+p^{a t m}\right]\right)=\left(g \eta+p^{a t m}\right) \partial_{x}\left(h_{\alpha} u_{\alpha}\right) \\
& +h_{\alpha} \partial_{x} q_{\alpha} u_{\alpha}+q_{\alpha+1 / 2}\left(u_{\alpha} \partial_{x} z_{\alpha+1 / 2}-w_{\alpha}\right)-q_{\alpha-1 / 2}\left(u_{\alpha} \partial_{x} z_{\alpha-1 / 2}-w_{\alpha}\right) \\
& -\Gamma_{\alpha+1 / 2}\left[u_{\alpha}\left(\widetilde{u}_{\alpha+1 / 2}-\frac{u_{\alpha}}{2}\right)+w_{\alpha}\left(\widetilde{w}_{\alpha+1 / 2}-\frac{w_{\alpha}}{2}\right)\right] \\
& +\Gamma_{\alpha-1 / 2}\left[u_{\alpha}\left(\widetilde{u}_{\alpha-1 / 2}-\frac{u_{\alpha}}{2}\right)+w_{\alpha}\left(\widetilde{w}_{\alpha-1 / 2}-\frac{w_{\alpha}}{2}\right)\right] .
\end{aligned}
$$

If we replace $q_{\alpha}$ in the right hand side thanks to (3.14), we recover (4.22) up to the term

$$
\frac{H^{2}}{4 L^{2}} \sum_{\alpha=1}^{L} \partial_{x} u_{\alpha}\left[\Gamma_{\alpha+1 / 2}\left(\widetilde{\left(\partial_{x} u\right)_{\alpha+1 / 2}}-\frac{\partial_{x} u_{\alpha}}{2}\right)-\Gamma_{\alpha-1 / 2}\left(\widetilde{\left(\partial_{x} u\right)_{\alpha-1 / 2}}-\frac{\partial_{x} u_{\alpha}}{2}\right)\right] .
$$

This term turns out to be equal to

$$
\frac{H^{2}}{4 L^{2}} \sum_{\alpha=1}^{L} \Gamma_{\alpha+1 / 2}\left(\gamma_{\alpha+1 / 2}-\frac{1}{2}\right)\left(\partial_{x} u_{\alpha+1}-\partial_{x} u_{\alpha}\right)^{2} .
$$

It is added to the classical term

$$
-\sum_{\alpha=1}^{L} \Gamma_{\alpha+1 / 2}\left(\gamma_{\alpha+1 / 2}-\frac{1}{2}\right)\left(1+\left|\partial_{x} z_{b}\right|^{2}\right) \llbracket u \rrbracket_{\alpha+1 / 2}^{2} .
$$

As previously, the constraint (4.16) upon $\gamma_{\alpha+1 / 2}$ is necessary to ensure that (B.3b) is actually negative. Consequently, (B.3a) is positive. In order to prevent the energy from increasing, we take $\gamma_{\alpha+1 / 2}=\frac{1}{2}$ so that both terms vanish and (B.2) holds. 


\section{Some properties of the $\mathrm{LDNH}_{0}$ model}

\section{C.1 Derivation of $\mathrm{LDNH}_{0}$}

Under (3.4)-(3.8), there is no need for an additional equation like (4.11) as $w$ is layerwise constant. The conservative equations with source terms (3.9a-3.9c) are derived similarly to $\S$ 4.1.2.

As for the dynamic pressure $q$, Equation (3.15b) expresses the hypothesis of continuity for a $\mathbb{P}_{1}$ function and looks like a simplification of (3.14).

We mention that Equation (3.15a) which is the counterpart of (3.9f) in the $\mathbb{P}_{0}$ framework also holds in the hydrostatic context [14].

\section{C.2 Energy}

If we replace $\overline{\mathcal{K}}_{\alpha}$ by $\bar{K}_{\alpha}$ as for the $\mathrm{LDNH}_{1}$ model - see (4.20), the statement of Prop. 1 stands for the $\mathrm{LDNH}_{0}$ model. Let us multiply $(3.9 \mathrm{~b})$ by $u_{\alpha}$ and $(3.9 \mathrm{c})$ by $w_{\alpha}$ and sum the resulting equalities:

$$
\begin{aligned}
\partial_{t}\left(h_{\alpha} \bar{K}_{\alpha}\right)+ & \partial_{x}\left(h_{\alpha} u_{\alpha}\left[\bar{K}_{\alpha}+q_{\alpha}+g \eta+p^{a t m}\right]\right)=\left(g \eta+p^{a t m}\right) \partial_{x}\left(h_{\alpha} u_{\alpha}\right)+h_{\alpha} q_{\alpha} \partial_{x} u_{\alpha} \\
& +q_{\alpha+1 / 2}\left(u_{\alpha} \partial_{x} z_{\alpha+1 / 2}-w_{\alpha}\right)-q_{\alpha-1 / 2}\left(u_{\alpha} \partial_{x} z_{\alpha-1 / 2}-w_{\alpha}\right) \\
& -\Gamma_{\alpha+1 / 2}\left(u_{\alpha} \widetilde{u}_{\alpha+1 / 2}+w_{\alpha} \widetilde{w}_{\alpha+1 / 2}-\bar{K}_{\alpha}\right)+\Gamma_{\alpha-1 / 2}\left(u_{\alpha} \widetilde{u}_{\alpha-1 / 2}+w_{\alpha} \widetilde{w}_{\alpha-1 / 2}-\bar{K}_{\alpha}\right) .
\end{aligned}
$$

Given (3.15a) and (3.15b), the terms involving $q$ become

$$
q_{\alpha+1 / 2} \sum_{\beta=1}^{\alpha} \partial_{x}\left(h_{\beta} u_{\beta}\right)-q_{\alpha-1 / 2} \sum_{\beta=1}^{\alpha-1} \partial_{x}\left(h_{\beta} u_{\beta}\right)
$$

whose sum over $\alpha$ vanishes. Then summing (C.1) leads to

$$
\begin{aligned}
\partial_{t}\left(\sum_{\alpha=1}^{L} h_{\alpha} \bar{K}_{\alpha}\right)+\partial_{x}\left(\sum_{\alpha=1}^{L} h_{\alpha} u_{\alpha}\left(\bar{K}_{\alpha}+q_{\alpha}+g \eta+p^{a t m}\right)\right) & =-\left(g \eta+p^{a t m}\right) \partial_{t} H+q_{1 / 2} \partial_{t} z_{b} \\
& -\sum_{\alpha=1}^{L} \Gamma_{\alpha+1 / 2}\left[u_{\alpha}\left(\widetilde{u}_{\alpha+1 / 2}-\frac{u_{\alpha}}{2}\right)+w_{\alpha}\left(\widetilde{w}_{\alpha+1 / 2}-\frac{w_{\alpha}}{2}\right)\right] \\
& -\Gamma_{\alpha-1 / 2}\left[u_{\alpha}\left(\widetilde{u}_{\alpha-1 / 2}-\frac{u_{\alpha}}{2}\right)+w_{\alpha}\left(\widetilde{w}_{\alpha-1 / 2}-\frac{w_{\alpha}}{2}\right)\right] .
\end{aligned}
$$

We conclude as previously by showing that the last terms are negative under (4.16).

\section{References}

[1] N. Ä̈ssiouene, M.-O. Bristeau, E. Godlewski, and J. Sainte-Marie. A combined finite volume-finite element scheme for a dispersive shallow water system. Netw. Heterog. Media, 11(1):1-27, 2016.

[2] N. Aïssiouene, M.-O. Bristeau, E. Godlewski, and J. Sainte-Marie. A robust and stable numerical scheme for a depth-averaged Euler system. hal-01162109, submitted.

[3] E. Audusse. A multilayer Saint-Venant model: derivation and numerical validation. Discrete Contin. Dyn. Syst. Ser. B, 5(2):189-214, 2005.

[4] E. Audusse, F. Bouchut, M.-O. Bristeau, R. Klein, and B. Perthame. A fast and stable well-balanced scheme with hydrostatic reconstruction for shallow water flows. SIAM J. Sci. Comput., 25(6):2050-2065, 2004. 
[5] E. Audusse, M.-O. Bristeau, B. Perthame, and J. Sainte-Marie. A multilayer Saint-Venant system with mass exchanges for Shallow Water flows. Derivation and numerical validation. ESAIM:Math. Model. Numer. Anal., 45(01):169-200, 2011.

[6] Y. Bai and K.F. Cheung. Dispersion and nonlinearity of multi-layer non-hydrostatic free-surface flow. J. Fluid Mech., 726:226-260, 2013.

[7] Y. Bai and K.F. Cheung. Dispersion and kinematics of multi-layer non-hydrostatic models. Ocean Model., 92:11-27, 2015.

[8] J.L. Bona, M. Chen, and J.-C. Saut. Boussinesq equations and other systems for small-amplitude long waves in nonlinear dispersive media. I: Derivation and linear theory. J. Nonlinear Sci., 12(4):283-318, 2002.

[9] J.L. Bona, M. Chen, and J.-C. Saut. Boussinesq equations and other systems for small-amplitude long waves in nonlinear dispersive media: II. The nonlinear theory. Nonlinearity, 17(3):925, 2004.

[10] P. Bonneton, F. Chazel, D. Lannes, F. Marche, and M. Tissier. A splitting approach for the fully nonlinear and weakly dispersive Green-Naghdi model. J. Comput. Phys., 230(4):1479-1498, 2011.

[11] F. Bouchut and M. Westdickenberg. Gravity driven shallow water models for arbitrary topography. Commun. Math. Sci., 2(3):359-389, 2004.

[12] F. Bouchut and V. Zeitlin. A robust well-balanced scheme for multi-layer shallow water equations. Discrete Contin. Dyn. Syst. Ser. B, 13(4):739-758, 2010.

[13] J. Boussinesq. Théorie des ondes et des remous qui se propagent le long d'un canal rectangulaire horizontal, en communiquant au liquide contenu dans ce canal des vitesses sensiblement pareilles de la surface au fond. J. Math. Pures Appl., pages 55-108, 1872.

[14] M.-O. Bristeau, B. Di Martino, C. Guichard, and J. Sainte-Marie. Layer-averaged Euler and NavierStokes equations. Commun. Math. Sci., 15(5):1221-1246, 2017.

[15] M.-O. Bristeau and J. Sainte-Marie. Derivation of a non-hydrostatic shallow water model; Comparison with Saint-Venant and Boussinesq systems. Discrete Contin. Dyn. Syst. Ser. B, 10(4), 2008.

[16] A. Castro and D. Lannes. Fully nonlinear long-wave models in the presence of vorticity. J. Fluid Mech., 759:642-675, 2014.

[17] V. Casulli. A semi-implicit finite difference method for non-hydrostatic, free-surface flows. Int. J. Numer. Methods Fluids, 30(4):425-440, 1999.

[18] V. Casulli and G.S. Stelling. Numerical simulation of 3d quasi-hydrostatic, free-surface flows. J. Hydrol. Eng., 124(7):678-686, 1998.

[19] R. Cienfuegos, E. Barthelemy, and P. Bonneton. A fourth-order compact finite volume scheme for fully nonlinear and weakly dispersive Boussinesq-type equations. Part I: model development and analysis. Int. J. Numer. Methods Fluids, 51(11):1217-1253, 2006.

[20] G. Corestein, E. Bladé, L. Cea, Á. Lara, E. Escolano, and A. Coll. Iber, a river dynamics simulation tool. In Proc. GiD Conference, 2010.

[21] Danish Hydrological Institute. MIKE HYDRO River, 2015.

[22] A. Barré de Saint-Venant. Théorie du mouvement non permanent des eaux, avec application aux crues des rivières et à l'introduction des marées dans leurs lits. C. R. Acad. Sci., 73:237-240, 1871.

[23] A. Decoene and J.-F. Gerbeau. Sigma transformation and ALE formulation for three-dimensional free surface flows. Int. J. Numer. Methods Fluids, 59(4):357-386, 2009. 
[24] E.D. Fernández-Nieto, E.H. Koné, and T. Chacón-Rebollo. A multilayer method for the hydrostatic Navier-Stokes equations: a particular weak solution. J. Sci. Comput., 60(2):408-437, 2014.

[25] E.D. Fernández-Nieto, E.H. Koné, T. Morales de Luna, and R. Bürger. A multilayer shallow water system for polydisperse sedimentation. J. Comput. Phys., 238:281-314, 2013.

[26] S. Ferrari and F. Saleri. A new two-dimensional Shallow Water model including pressure effects and slow varying bottom topography. ESAIM: Math. Model. Numer. Anal., 38(2):211-234, 2004.

[27] J.-F. Gerbeau and B. Perthame. Derivation of viscous Saint-Venant system for laminar shallow water; numerical validation. Discrete Contin. Dyn. Syst. Ser. B, 1(1):89-102, 2001.

[28] M.F. Gobbi, J.T. Kirby, and G.E. Wei. A fully nonlinear Boussinesq model for surface waves. Part 2. Extension to $\mathcal{O}\left((k h)^{4}\right)$. J. Fluid Mech., 405:181-210, 2000.

[29] A.E. Green and P.M. Naghdi. A derivation of equations for wave propagation in water of variable depth. J. Fluid Mech., 78(02):237-246, 1976.

[30] J.M. Greenberg and A.-Y. Leroux. A well-balanced scheme for the numerical processing of source terms in hyperbolic equations. SIAM J. Numer. Anal., 33(1):1-16, 1996.

[31] F.H. Harlow and J.E. Welch. Numerical calculation of time-dependent viscous incompressible flow of fluid with free surface. Phys. Fluids, 8(12):2182, 1965.

[32] J.M. Hervouet and L. Van Haren. Telemac2d principle note 3.0. Technical report, EDF, 1996. HE4394052B.

[33] B.R. Hodges, B. Laval, and B.M. Wadzuk. Numerical error assessment and a temporal horizon for internal waves in a hydrostatic model. Ocean Model., 13(1):44-64, 2006.

[34] Hydrologic Engineering Center. HEC-RAS River Analysis System, manual version 5.0 edition, April 2015.

[35] J.T. Kirby. Boussinesq models and their application to coastal processes across a wide range of scales. J. Waterway, Port, Coastal, Ocean Eng., 142(6):03116005, 2016.

[36] D. Lannes. The Water Waves Problem: Mathematical Analysis and Asymptotics, volume 188. Amer. Math. Soc., 2013.

[37] D. Lannes and P. Bonneton. Derivation of asymptotic two-dimensional time-dependent equations for surface water wave propagation. Phys. Fluids, 21(1):016601, 2009.

[38] P.J. Lynett and P.L.-F. Liu. Linear analysis of the multi-layer model. Coastal Eng., 51(5):439-454, 2004.

[39] P.A. Madsen, R. Murray, and O.R. Sørensen. A new form of the Boussinesq equations with improved linear dispersion characteristics. Coastal Eng., 15(4):371-388, 1991.

[40] P.A. Madsen and O.R. Sørensen. A new form of the Boussinesq equations with improved linear dispersion characteristics. Part 2. A slowly-varying bathymetry. Coastal Eng., 18(3-4):183-204, 1992.

[41] F. Marche. Derivation of a new two-dimensional viscous shallow water model with varying topography, bottom friction and capillary effects. Eur. J. Mech. B Fluids, 26(1):49-63, 2007.

[42] E. Miglio, A. Quarteroni, and F. Saleri. Finite element approximation of quasi-3D shallow water equations. Comput. Methods Appl. Mech. Engrg., 174(3):355-369, 1999.

[43] O. Nwogu. Alternative form of Boussinesq equations for nearshore wave propagation. J. Waterway, Port, Coastal, Ocean Eng., 119(6):618-638, 1993.

[44] D.H. Peregrine. Long waves on a beach. J. Fluid Mech., 27(04):815-827, 1967. 
[45] B. Perthame and C. Simeoni. A kinetic scheme for the Saint-Venant system with a source term. Calcolo, 38(4):201-231, 2001.

[46] N.A. Phillips. A coordinate system having some special advantages for numerical forecasting. J. Meteorol., 14(2):184-185, 1957.

[47] G. Richard and S. Gavrilyuk. A new model of roll waves: comparison with Brock's experiments. J. Fluid Mech., 698:374-405, 2012.

[48] G. Richard and S. Gavrilyuk. The classical hydraulic jump in a model of shear shallow-water flows. $J$. Fluid Mech., 725:492-521, 2013.

[49] J. Sainte-Marie. Vertically averaged models for the free surface non-hydrostatic Euler system: derivation and kinetic interpretation. Math. Models Methods Appl. Sci, 21(03):459-490, 2011.

[50] F. Serre. Contribution à l'étude des écoulements permanents et variables dans les canaux. La Houille Blanche, (6):830-872, 1953.

[51] P.K. Stansby and J.G. Zhou. Shallow-water flow solver with non-hydrostatic pressure: 2D vertical plane problems. Int. J. Numer. Methods Fluids, 28(3):541-563, 1998.

[52] M.E. Vásquez-Cendón. Improved treatment of source terms in upwind schemes for the shallow water equations in channels with irregular geometry. J. Comput. Phys., 148(2):497-526, 1999.

[53] G.E. Wei, J.T. Kirby, S.T. Grilli, and R. Subramanya. A fully nonlinear Boussinesq model for surface waves. I: Highly nonlinear unsteady waves. J. Fluid Mech., 294:71-92, 1995.

[54] J.M. Witting. A unified model for the evolution nonlinear water waves. J. Comput. Phys., 56(2):203-236, 1984.

[55] Y. Yamazaki, Z. Kowalik, and K.F. Cheung. Depth-integrated, non-hydrostatic model for wave breaking and run-up. Int. J. Numer. Methods Fluids, 61(5):473-497, 2009.

[56] M. Zijlema, G. Stelling, and P. Smit. SWASH: An operational public domain code for simulating wave fields and rapidly varied flows in coastal waters. Coastal Eng., 58(10):992-1012, 2011.

[57] M. Zijlema and G.S. Stelling. Further experiences with computing non-hydrostatic free-surface flows involving water waves. Int. J. Numer. Methods Fluids, 48(2):169-197, 2005. 